\title{
Homogenization of diffusion problems with a nonlinear interfacial resistance
}

\author{
Patrizia Donato@ and Kim Hang Le Nguyen
}

\begin{abstract}
In this paper, we consider a stationary heat problem on a twocomponent domain with an $\varepsilon$-periodic imperfect interface, on which the heat flux is proportional via a nonlinear function to the jump of the solution, and depends on a real parameter $\gamma$. Homogenization and corrector results for the corresponding linear case have been proved in Donato et al. (J Math Sci 176(6):891-927, 2011), by adapting the periodic unfolding method [see (Cioranescu et al. SIAM J Math Anal 40(4):1585-1620, 2008), (Cioranescu et al. SIAM J Math Anal 44(2):718-760, 2012), (Cioranescu et al. Asymptot Anal 53(4):209-235, 2007)] to the case of a twocomponent domain. Here, we first prove, under natural growth assumptions on the nonlinearities, the existence and the uniqueness of a solution of the problem. Then, we study, using the periodic unfolding method, its asymptotic behavior when $\varepsilon \rightarrow 0$. In order to describe the homogenized problem, we complete some convergence results of Donato et al. ( $\mathrm{J}$ Math Sci 176(6):891-927, 2011) concerning the unfolding operators and we investigate the limit behaviour of the unfolded Nemytskii operators associated to the nonlinear terms. According to the values of the parameter $\gamma$ we have different limit problems, for the cases $\gamma<-1, \gamma=-1$ and $\gamma \in]-1,1]$. The most relevant case is $\gamma=-1$, where the homogenized matrix differs from that of the linear case, and is described in a more complicated way, via a nonlinear function involving the correctors.
\end{abstract}

Mathematics Subject Classification. 35B27, 35J65, 82B24.

Keywords. Periodic homogenization, Elliptic equations with jump, Nonlinear interface conditions.

\section{Introduction}

In the present paper we investigate the thermal conduction in a composite material of two components in the presence of a nonlinear interfacial thermal resistance, which is described by nonlinear transmission conditions on the interface. 
More precisely, we study the periodic homogenization of the following problem:

$$
\begin{cases}-\operatorname{div}\left(A^{\varepsilon} \nabla u_{1}^{\varepsilon}\right)+h_{1}^{\varepsilon}\left(x, u_{1}^{\varepsilon}\right)=f & \text { in } \Omega_{1}^{\varepsilon}, \\ -\operatorname{div}\left(A^{\varepsilon} \nabla u_{2}^{\varepsilon}\right)+h_{2}^{\varepsilon}\left(x, u_{2}^{\varepsilon}\right)=f & \text { in } \Omega_{2}^{\varepsilon}, \\ A^{\varepsilon} \nabla u_{1}^{\varepsilon} \cdot n_{1}^{\varepsilon}=-A^{\varepsilon} \nabla u_{2}^{\varepsilon} \cdot n_{2}^{\varepsilon} & \text { on } \Gamma^{\varepsilon}, \\ -A^{\varepsilon} \nabla u_{1}^{\varepsilon} \cdot n_{1}^{\varepsilon}=\varepsilon^{\gamma+1} h^{\varepsilon}\left(x, u_{1}^{\varepsilon}-u_{2}^{\varepsilon}\right) & \text { on } \Gamma^{\varepsilon}, \\ u_{i}^{\varepsilon}=0 & \text { on } \partial \Omega \cap \partial \Omega_{i}^{\varepsilon}, i=1,2,\end{cases}
$$

where $\Omega=\Omega_{1}^{\varepsilon} \cup \bar{\Omega}_{2}^{\varepsilon}$ is a two-component domain, $\gamma \in \mathbb{R}, f \in L^{2}(\Omega), n_{i}^{\varepsilon}$ is the unit outward normal to $\Omega_{i}^{\varepsilon}$, for $i=1,2$.

The two components are open disjoint subsets of the open bounded set $\Omega \in \mathbb{R}^{n}$, separated by a common boundary $\Gamma^{\varepsilon}$. The component $\Omega_{2}^{\varepsilon}$ is the (non connected) intersection of $\Omega$ with the union of the $\varepsilon$-periodic translated sets of $\varepsilon Y_{2}$, where $\overline{Y_{2}}$ is contained in the reference periodicity cell $Y$. The other one $\Omega_{1}^{\varepsilon}=\Omega \backslash \bar{\Omega}_{2}^{\varepsilon}$ is connected (see Fig. 1 in the next section).

We assume that the thermal conductivity of material is given by $A^{\varepsilon}(x)=$ $A(x / \varepsilon)$, the matrix $A$ being $Y$-periodic, uniformly elliptic and bounded. The nonlinear terms $h_{i}^{\varepsilon}$ in the equation are defined by $h_{i}^{\varepsilon}(x, s)=h_{i}(x / \varepsilon, s)$ for $i=1,2$, where $h_{1}$ and $h_{2}$ are Carathéodory functions satisfying some natural growth conditions.

In the boundary conditions in (1.1) the nonlinear term $h^{\varepsilon}$ is given by $h^{\varepsilon}(x, s)=h(x / \varepsilon, s / \varepsilon)$, so that the heat flux through the interface is continuous, and proportional via a nonlinear function $h$ to the jump of the temperature. This means that the rate of heat transfer across the interfacial barrier is proportional to the temperature difference by a nonlinear rule. Actually, according to experimental results, in most cases, the flux on the interface is not a linear function of the difference of temperature between two components (see for instance [10]). This nonlinear condition generalizes the linear one of the well-known Barenblatt's model (see [5]).

Let us point out that in this work we allow the holes to meet the boundary of the domain $\Omega$ and then $\Omega_{1}^{\varepsilon}, \Omega_{2}^{\varepsilon}, \Gamma^{\varepsilon}$ are somewhat different from those considered in the linear case [26], where $\Omega_{2}^{\varepsilon}$ only contains the holes inside $\Omega$.

Let us also mention that nonlinear interface conditions also arise in some evolution problem modeling the electrical conduction in biological tissues, in the presence of an interfacial resistance property of the cell membranes, see $[2]$.

In the present work, we apply the periodic unfolding method to get the asymptotic behavior of the nonlinear problem (1.1) as $\varepsilon$ tends to zero. In [26] the authors have recently adapted this method to the case of two-component domains by considering two unfolding operators: the first one, denoted $\mathcal{T}_{1}^{\varepsilon}$, acts on functions defined in $\Omega_{1}^{\varepsilon}$ and was originally denoted $\mathcal{T}_{\varepsilon}^{*}$ in the literature for perforated domains $[13,15,16]$; the second one, appropriate to case of twocomponent domains and denoted $\mathcal{T}_{2}^{\varepsilon}$, concerns functions defined in $\Omega_{2}^{\varepsilon}$. They maps functions defined on the oscillating domains $\Omega_{1}^{\varepsilon}, \Omega_{2}^{\varepsilon}$ into functions defined 
on the fixed ones $\Omega \times Y_{1}$ and $\Omega \times Y_{2}$, respectively. In particular, the relationship between these two operators and the properties of their traces on the common boundaries was also studied. These results were applied in order to recover and improve the homogenization and corrector results obtained by S. Monsurrò and P. Donato in $[20,25,36]$.

The periodic unfolding method was introduced for the first time by Cioranescu et al. [11]. It gives an elementary proof for the classical periodic homogenization problem originally considered in [6] (see also [14,40]) and corrector results under natural assumptions (see [12] for a comprehensive presentation). It was extended to the case of perforated domains in $[13,15,16]$ and allows to avoid using the extension operators, so that no condition for the existence of such extensions is required as traditionally done in the literature (see $[17,18]$ ).

Let us mention that if $\left\{v_{n}\right\}$ is a bounded sequence of $L^{p}(\Omega)$, then the weak $L^{p}$-convergence of the unfolded sequence $\left\{\mathcal{T}_{\varepsilon}\left(v_{n}\right)\right\}$ is equivalent to the two-scale convergence of $\left\{v_{n}\right\}$. We recall that the notion of two-scale convergence has been introduced by Nguetseng in [38] and further developed by Allaire in [1] with applications to periodic homogenization.

In the first part of the present work, we show an existence and uniqueness result of the weak solution of problem (1.1), using the classical MintyBrowder theorem. Then, we complete the study done in [26] with some new results needed here for our nonlinear problem, including some convergence results concerning the limit of the unfolded nonlinear terms in the variational formulation of the problem (see Proposition 4.7 for more details). In the second part, we study the homogenization of problem (1.1) for the different values of $\gamma \leq 1$

Let us briefly describe the difficulties due to the presence of the nonlinear jump and the nonlinear function $h_{2}$. The main difficulty is related to the nonlinearity in the boundary condition, which gives rise to a term of the form

$$
\begin{aligned}
\varepsilon^{\gamma+1} \int_{\Gamma^{\varepsilon}} h^{\varepsilon}\left(x, u_{1}^{\varepsilon}-u_{2}^{\varepsilon}\right)\left(v_{1}-v_{2}\right) d \sigma_{x}= & \varepsilon^{\gamma} \frac{1}{|Y|} \int_{\Omega \times \Gamma} h\left(y, \frac{\mathcal{T}_{b}^{\varepsilon}\left(u_{1}^{\varepsilon}-u_{2}^{\varepsilon}\right)}{\varepsilon}\right) \\
& \times\left(\mathcal{T}_{1}^{\varepsilon}\left(v_{1}\right)-\mathcal{T}_{2}^{\varepsilon}\left(v_{2}\right)\right) d x d \sigma_{y} .
\end{aligned}
$$

In view of the results in [26], we prove that this term goes to zero for $\gamma>-1$, while for $\gamma<-1$ the choice of test functions without jump, which makes the term vanish, is enough to pass to the limit. This implies that the homogenized matrix $A_{\gamma}^{0}$ is the same as that of the linear case with jump studied before in the literature.

The case $\gamma=-1$ presents a major difficulty since we have only the weak convergence of $\mathcal{T}_{b}^{\varepsilon}\left(u_{1}^{\varepsilon}-u_{2}^{\varepsilon}\right) / \varepsilon$ in $L^{2}(\Omega \times \Gamma)$ so that we need to identify the limit of the unfolded boundary term. To do that, we use the Minty method for monotone operators together with a suitable choice of sequence of test functions and we obtain a boundary term in the limit problem. The homogenized matrix is described in a more complicated way, since it is expressed via a non- 
linear function involving the correctors, as shown by Corollary 5.5. Moreover, in this case, the uniqueness of the limit problem needs to be proved.

Concerning the nonlinear function $h_{2}$, it leads to pass to the limit in terms of the form

$$
\int_{\Omega_{2}^{\varepsilon}} h_{2}^{\varepsilon}\left(x, u_{2}^{\varepsilon}\right) v_{2} d x=\frac{1}{|Y|} \int_{\Omega \times Y_{2}} h_{2}\left(y, \mathcal{T}_{2}^{\varepsilon}\left(u_{2}^{\varepsilon}\right)\right) \mathcal{T}_{2}^{\varepsilon}\left(v_{2}\right) d x d y,
$$

where the weak convergence of $\mathcal{T}_{2}^{\varepsilon}\left(u_{2}^{\varepsilon}\right)$ in $L^{2}\left(\Omega, H^{1}\left(Y_{2}\right)\right)$ proved in [26] is not sufficient to pass to the limit. To overcome this difficulty we improve here the previous results proving that this convergence is actually strong for $\gamma<1$ (see Theorem 4.5). For the case $\gamma=1$, the assumption that $h_{2} \equiv 0$ is required since we cannot improve the weak convergence result of $\mathcal{T}_{2}^{\varepsilon}\left(u_{2}^{\varepsilon}\right)$. Some results for this case have been announced in [41] where also $\Omega_{2}^{\varepsilon}$ is supposed connected.

The pioneer paper on this subject was proposed by Auriault and Ene in [4] for the linear case, using the multiple scale method. In $[20,21,25,36,37]$, the linear case $\left(h_{1}=h_{2}=0\right.$ and $h(y, s)=s \tilde{h}(y)$, where $\tilde{h}(y)$ is an $Y$-periodic positive bounded function) was studied using the Tartar method and assuming that the holes do not meet the boundary.

For similar homogenization problems of elliptic type we refer the reader to $[9,27,28,30,33,34,39]$ and for related parabolic problems [22-24,29,31], together with the references therein. For the homogenization of linear and quasilinear elliptic problems with a nonlinear Robin condition containing a nonlinear term with the same growth as that in the boundary condition considered in the present paper, we refer to $[8,16]$, respectively, where the periodic unfolding method was used.

The paper is organized as follows:

In Sect. 2, we introduce the problem together with the assumptions concerning the functions $h, h_{1}$ and $h_{2}$.

Section 3 is devoted to the existence and uniqueness of a weak solution of problem (1.1).

In Section 4, we recall some properties of the unfolding operators introduced in $[13,15,26]$ and give some new convergence results.

Finally, in Sect. 5 we state and prove the homogenization results for the different case of the parameter, $\gamma<-1, \gamma=-1$ and $\gamma \in]-1,1]$.

\section{Statement of the problem}

\subsection{Notations}

In this section we introduce the main notations and the two-component domain.

In $\mathbb{R}^{n}(n \geq 2)$, let $\Omega$ be an open bounded set with a Lipschitz continuous boundary $\partial \Omega$ and $Y:=\prod_{i=1}^{n}\left[0, l_{i}\right.$ [ be a reference cell, with $l_{i}>0, i=1, \ldots, n$. We assume that $Y_{1}$ and $Y_{2}$ are two disjoint connected open subsets of $Y$ such that $Y_{2} \neq \varnothing, \bar{Y}_{2} \subset Y$ and $Y=Y_{1} \cup \bar{Y}_{2}$, with a common boundary $\Gamma=\partial Y_{2}$ Lipschitz continuous (see Fig. 1). 


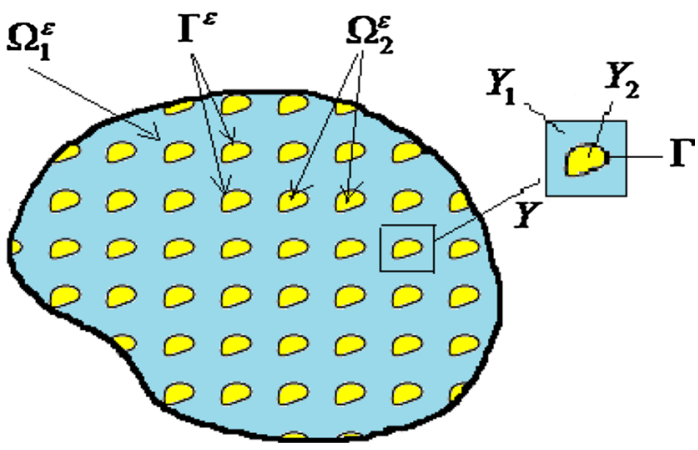

Figure 1. The two-component composite

Remark 2.1. For the sake of simplicity, in this paper we assume that $Y_{2}$ is connected. Actually the results given here can be extended to the case where $Y_{2}$ has a finite number of connected components, in the spirit of Theorems 2.21 and 3.11 in [26].

Throughout this paper, $\varepsilon$ will take its values in a positive real sequence which tends to zero and $c$ is a constant independent of $\varepsilon$.

For almost every $z \in \mathbb{R}^{n}$, we use $[z]_{Y}$ to denote its integer part $\left(k_{1} l_{1}, \ldots, k_{n} l_{n}\right), k_{i} \in \mathbb{Z}$ for $i=1, \ldots, n$, such that $z-[z]_{Y} \in Y$ and set $\{z\}_{Y}=z-[z]_{Y}$.

Then, we have

$$
x=\varepsilon\left(\left[\frac{x}{\varepsilon}\right]_{Y}+\left\{\frac{x}{\varepsilon}\right\}_{Y}\right) \quad \text { for a.e. } x \in \mathbb{R}^{n} .
$$

Let us introduce the two-component domain as follows (see Fig. 1):

- for any $k=\left(k_{1}, \ldots, k_{n}\right) \in \mathbb{Z}^{n}, k_{l}=\left(k_{1} l_{1}, \ldots, k_{n} l_{n}\right)$ and

$$
Y^{k}=k_{l}+Y, \quad Y_{i}^{k}=k_{l}+Y_{i}, \quad i=1,2,
$$

- $K_{\varepsilon}=\left\{k \in \mathbb{Z}^{n} \mid \varepsilon Y_{i}^{k} \cap \Omega \neq \varnothing, i=1,2\right\}$ and

$$
\Omega_{1}^{\varepsilon}=\Omega \backslash \bigcup_{k \in K_{\varepsilon}} \varepsilon \overline{Y_{2}^{k}}, \quad \Gamma^{\varepsilon}=\partial \Omega_{1}^{\varepsilon} \cap \Omega, \quad \Omega_{2}^{\varepsilon}=\Omega \backslash \overline{\Omega_{1}^{\varepsilon}} .
$$

We allow here the holes to meet the boundary of the domain $\Omega$. This makes the domains $\Omega_{1}^{\varepsilon}, \Omega_{2}^{\varepsilon}$ and $\Gamma^{\varepsilon}$ somewhat different from themselves in our last paper [26] for the linear case, where $\Omega_{2}^{\varepsilon}$ only contains the holes inside $\Omega$. Here the domain $\Omega_{2}^{\varepsilon}$ is the intersection of $\Omega$ and the union of the $\varepsilon Y$-periodic translated sets of the hole $\varepsilon Y_{2}$. Consequently, the interface $\Gamma^{\varepsilon}$ is bigger than the corresponding in [26].

The following notations are also used in the sequel:

- $\theta_{i}=\frac{\left|Y_{i}\right|}{|Y|}, i=1,2$,

- $\widetilde{u}$ : the zero extension to the whole $\Omega$ of a function $u$ defined on $\Omega_{1}^{\varepsilon}$ or $\Omega_{2}^{\varepsilon}$,

- $\chi_{\omega}$ : the characteristic function of each open set $\omega$ of $\mathbb{R}^{n}$, 
- $\mathcal{M}_{\omega}(f):=\frac{1}{|\omega|} \int_{\omega} f d x$, for any open set $\omega$ of $\mathbb{R}^{n}$ and for any $f \in L^{1}(\omega)$,

- $M(\alpha, \beta, \mathcal{O})$ : the set of the $n \times n$ matrix-valued functions $A$ in $\left(L^{\infty}(\mathcal{O})\right)^{n^{2}}$ such that, for any $\lambda \in \mathbb{R}^{n}$,

$$
\begin{cases}(A(x) \lambda, \lambda) \geq \alpha|\lambda|^{2} & \text { a.e. in } \mathcal{O} \\ |A(x) \lambda| \leq \beta|\lambda| & \text { a.e. in } \mathcal{O}\end{cases}
$$

for $\alpha, \beta \in \mathbb{R}, \quad 0<\alpha \leq \beta$.

\subsection{The problem}

Our goal is to describe the asymptotic behavior, as $\varepsilon \rightarrow 0$, of the following problem:

$$
\begin{cases}-\operatorname{div}\left(A^{\varepsilon} \nabla u_{1}^{\varepsilon}\right)+h_{1}^{\varepsilon}\left(x, u_{1}^{\varepsilon}\right)=f & \text { in } \Omega_{1}^{\varepsilon}, \\ -\operatorname{div}\left(A^{\varepsilon} \nabla u_{2}^{\varepsilon}\right)+h_{2}^{\varepsilon}\left(x, u_{2}^{\varepsilon}\right)=f & \text { in } \Omega_{2}^{\varepsilon}, \\ A^{\varepsilon} \nabla u_{1}^{\varepsilon} \cdot n_{1}^{\varepsilon}=-A^{\varepsilon} \nabla u_{2}^{\varepsilon} \cdot n_{2}^{\varepsilon} & \text { on } \Gamma^{\varepsilon}, \\ -A^{\varepsilon} \nabla u_{1}^{\varepsilon} \cdot n_{1}^{\varepsilon}=\varepsilon^{\gamma+1} h^{\varepsilon}\left(x, u_{1}^{\varepsilon}-u_{2}^{\varepsilon}\right) & \text { on } \Gamma^{\varepsilon}, \\ u_{i}^{\varepsilon}=0 & \text { on } \partial \Omega \cap \partial \Omega_{i}^{\varepsilon}, \quad i=1,2,\end{cases}
$$

where the coefficient matrix $A^{\varepsilon}$ and the nonlinear terms $h^{\varepsilon}, h_{i}^{\varepsilon}$ are given by

$$
\left\{\begin{array}{l}
A^{\varepsilon}(x)=A\left(\frac{x}{\varepsilon}\right) \\
h^{\varepsilon}(x, s)=h\left(\frac{x}{\varepsilon}, \frac{s}{\varepsilon}\right) \\
h_{i}^{\varepsilon}(x, s)=h_{i}\left(\frac{x}{\varepsilon}, s\right) \text { for } i=1,2
\end{array}\right.
$$

with $A, h_{1}$ and $h_{2}$ satisfying assumptions (2.4)-(2.5) below.

The linear case, where $h_{1}=h_{2}=0$ and $h$ is of the form $h(y, s)=s \tilde{h}(y)$, was studied, under the assumption that the holes do not intersect the exterior boundary, by Tartar's method in $[25,36]$, by two-scale method in [28] and recently by unfolding in [26].

Here we investigate a more general situation, where a nonlinear function of the jump of the solution appears on the rapidly oscillating interface together with the presence of the two nonlinear zero-order terms $h_{1}$ and $h_{2}$.

Throughout the paper, we assume that

$$
\left\{\begin{array}{l}
\gamma \leq 1, \quad f \in L^{2}(\Omega), \\
A \in M(\alpha, \beta, Y), \quad A \text { is } Y \text {-periodic, }
\end{array}\right.
$$

and

$$
\left\{\begin{array}{l}
h \text { satisfies }\left(\mathcal{H}_{1}\right)-\left(\mathcal{H}_{3}\right), \\
h_{1} \text { satisfies }\left(\mathcal{H}_{1}\right) \text { and }\left(\mathcal{H}_{4}\right), \\
h_{2} \text { satisfies }\left(\mathcal{H}_{1}\right) \text { and }\left(\mathcal{H}_{4}^{\prime}\right),
\end{array}\right.
$$

for some $\alpha, \beta \in \mathbb{R}, 0<\alpha \leq \beta$ and assumptions $\left(\mathcal{H}_{1}\right)-\left(\mathcal{H}_{4}\right)$ shown below.

Furthermore, the homogenization results obtained in Sect. 5 depend on the value of $\gamma$. Then, for the reader's convenience, we list the different cases and the corresponding assumptions as follows: 


$$
\begin{cases}h \text { or } h_{2} \text { is strictly increasing } & \text { if } \gamma \neq \pm 1 \\ h \text { is strictly increasing } & \text { if } \gamma=-1 \\ h \text { is strictly increasing and } h_{2} \equiv 0 & \text { if } \gamma=1 .\end{cases}
$$

Let us introduce the hypotheses related to the two-variable functions $h, h_{1}$ and $h_{2}$.

Assumption $\mathcal{H}_{1}$ : The function $g(y, s): \mathbb{R}^{n} \times \mathbb{R} \longrightarrow \mathbb{R}$ satisfies assumption $\left(\mathcal{H}_{1}\right)$ iff

1. $g$ is a Carathéodory function,

2. $g(\cdot, s)$ is a (measurable) $Y$-periodic function for all $s \in \mathbb{R}$,

3. $g(y, \cdot)$ is an increasing function in $C^{1}(\mathbb{R})$ s.t. $g(y, 0)=0$ for all $y \in \mathbb{R}^{n}$.

Assumption $\mathcal{H}_{2}$ : The function $g(y, s): Y \times \mathbb{R} \longrightarrow \mathbb{R}$ satisfies assumption $\left(\mathcal{H}_{2}\right)$ iff there exists a constant $c>0$ and an exponent $q$, with

$$
1 \leq q<2 \text { if } n=2,3 \text { and } 1 \leq q<\frac{n}{n-2} \text { if } n>3
$$

such that

$$
\left|\frac{\partial g}{\partial s}(y, s)\right| \leq c\left(1+|s|^{q-1}\right) \quad \text { for a.e. } y \in Y \text { and for all } s \in \mathbb{R} .
$$

Assumption $\mathcal{H}_{3}$ : The function $g(y, s): Y \times \mathbb{R} \longrightarrow \mathbb{R}$ satisfies assumption $\left(\mathcal{H}_{3}\right)$ iff there exists a constant $c>0$ such that

$$
s g(y, s) \geq c|s|^{2} \quad \text { for a.e. } y \in Y \text { and for all } s \in \mathbb{R} \text {. }
$$

Assumption $\mathcal{H}_{4}$ : The function $g(y, s): Y \times \mathbb{R} \longrightarrow \mathbb{R}$ satisfies assumption $\left(\mathcal{H}_{4}\right)$ (resp. $\left.\left(\mathcal{H}_{4}^{\prime}\right)\right)$ iff there exists a constant $c>0$ and an exponent $p_{1}$ (resp. $p_{2}$ ), with

$$
1 \leq p_{1}<+\infty \text { if } n=2 \quad \text { and } \quad 1 \leq p_{1} \leq \frac{n+2}{n-2} \text { if } n>2
$$

(resp. $\left.1 \leq p_{2} \leq \min \left\{2, \frac{n+2}{n-2}\right\}\right)$ such that

$$
\left|\frac{\partial g}{\partial s}(y, s)\right| \leq c\left(1+|s|^{p_{i}-1}\right) \quad \text { for a.e. } y \in Y \text { and for all } s \in \mathbb{R} \text {. }
$$

Remark 2.2. It is easy to check that

(i) the function $g(y, s)=\xi(y)\left(s+|s|^{q-1} s\right)\left(\operatorname{resp} . \xi(y)\left(s+|s|^{p_{1}-1} s\right)\right)$ satisfies assumptions $\left(\mathcal{H}_{1}\right)-\left(\mathcal{H}_{3}\right)$ (resp. $\left(\mathcal{H}_{1}\right),\left(\mathcal{H}_{3}\right)$ and $\left.\left(\mathcal{H}_{4}\right)\right)$ where $\xi(y)$ is any positive bounded measurable $Y$-periodic function,

(ii) if $g(y, s)$ satisfies assumption $\left(\mathcal{H}_{1}\right)$, then $s g(y, s) \geq 0$ for a.e. $y \in$ $Y$ and for all $s \in \mathbb{R}$,

(iii) if $g(y, s)$ satisfies assumptions $\left(\mathcal{H}_{1}\right)$ and $\left(\mathcal{H}_{2}\right)$, then for some $c>0$,

$$
|g(y, s)| \leq c\left(1+|s|^{q}\right) \quad \text { for a.e. } y \in Y \text { and for all } s \in \mathbb{R} \text {, }
$$

since, due to the fact that $g(y, 0)=0$ for all $y \in \mathbb{R}^{n}$, we have

$|g(y, s)|=\left|\int_{0}^{s} \frac{\partial g}{\partial \tau}(y, \tau) d \tau\right| \leq c\left|\int_{0}^{s}\left(1+|\tau|^{q-1}\right) d \tau\right| \leq c\left(|s|+|s|^{q}\right)$,

and 
- if $|s| \leq 1,|g(y, s)| \leq c\left(1+|s|^{q}\right)$,

- if $|s|>1,|g(y, s)| \leq 2 c|s|^{q}<2 c\left(1+|s|^{q}\right)$.

We now introduce the functional spaces adapted to our problem.

Definition 2.3. For every $\gamma \in \mathbb{R}$,

$$
H_{\gamma}^{\varepsilon} \doteq\left\{u=\left(u_{1}, u_{2}\right) \mid u_{1} \in V_{1}^{\varepsilon}, u_{2} \in V_{2}^{\varepsilon}\right\}
$$

equipped with the norm

$$
\|u\|_{H_{\gamma}^{\varepsilon}}^{2}=\left\|\nabla u_{1}\right\|_{L^{2}\left(\Omega_{1}^{\varepsilon}\right)}^{2}+\left\|\nabla u_{2}\right\|_{L^{2}\left(\Omega_{2}^{\varepsilon}\right)}^{2}+\varepsilon^{\gamma}\left\|u_{1}-u_{2}\right\|_{L^{2}\left(\Gamma^{\varepsilon}\right)}^{2},
$$

where $V_{i}^{\varepsilon} \doteq\left\{v \in H^{1}\left(\Omega_{i}^{\varepsilon}\right) \mid v=0\right.$ on $\left.\partial \Omega \cap \partial \Omega_{i}^{\varepsilon}\right\}, i=1,2$, endowed respectively with the norms

$$
\|v\|_{V_{1}^{\varepsilon}}=\|\nabla v\|_{L^{2}\left(\Omega_{1}^{\varepsilon}\right)}, \quad\|v\|_{V_{2}^{\varepsilon}}=\left(\|v\|_{L^{2}\left(\Omega_{2}^{\varepsilon}\right)}^{2}+\|\nabla v\|_{L^{2}\left(\Omega_{2}^{\varepsilon}\right)}^{2}\right)^{1 / 2} .
$$

Remark 2.4. $\quad$ (i) As observed in [13], the Lipschitz assumption of $\partial \Omega$ implies that for every open subset $\Omega_{0}$ of $\mathbb{R}^{n}$ such that $\Omega \subset \Omega_{0}$ and $\partial \Omega \cap \partial \Omega_{1}^{\varepsilon}=$ $\partial \Omega \cap \Omega_{0}$,

$$
V_{1}^{\varepsilon}=\left\{v \in H^{1}\left(\Omega_{1}^{\varepsilon}\right) \mid \exists v^{\prime} \in H^{1}\left(\Omega_{0}^{\varepsilon}\right), v^{\prime}=0 \text { in } \Omega_{0}^{\varepsilon} \backslash \overline{\Omega_{1}^{\varepsilon}} \text { and } v=\left.v^{\prime}\right|_{\Omega_{1}^{\varepsilon}}\right\},
$$

where $\Omega_{0}^{\varepsilon}=\Omega_{0} \backslash \bigcup_{k \in \mathbb{Z}^{n}} \varepsilon \overline{Y_{2}^{k}}$.

(ii) The norm $\|\cdot\|_{V_{1}^{\varepsilon}}$ is equivalent to $\|\cdot\|_{H^{1}\left(\Omega_{1}^{\varepsilon}\right)}$ by constants independent of $\varepsilon$ since the Poincaré inequality holds in the space $V_{1}^{\varepsilon}$ with a constant $c$ independent of $\varepsilon$, that is

$$
\|v\|_{L^{2}\left(\Omega_{1}^{\varepsilon}\right)} \leq c\|\nabla v\|_{L^{2}\left(\Omega_{1}^{\varepsilon}\right)} \quad \forall v \in V_{1}^{\varepsilon},
$$

(see Theorem 2.9 in [13] for more details).

The space $H_{\gamma}^{\varepsilon}$ defined above is not exactly the one introduced in [24$26,36]$, since as mentioned above, here the holes can meet the boundary of $\Omega$ and then $\Gamma_{\varepsilon}$ is somewhat different. Nevertheless, it is easy to check that the properties of the space $H_{\gamma}^{\varepsilon}$ proved in the papers quoted above are still true for this situation. In particular, the following proposition is still true.

Proposition 2.5. [24] There exist some positive constants $c, c_{1}$ and $c_{2}$, independent of $\varepsilon$, such that

$$
\|u\|_{H_{\gamma}^{\varepsilon}}^{2} \leq c\left(1+\varepsilon^{\gamma-1}\right)\|u\|_{V_{1}^{\varepsilon} \times V_{2}^{\varepsilon}}^{2} \quad \forall \gamma \in \mathbb{R}, \forall u \in H_{\gamma}^{\varepsilon}
$$

Moreover, if $\gamma \leq 1$

$$
c_{1}\|u\|_{V_{1}^{\varepsilon} \times V_{2}^{\varepsilon}}^{2} \leq\|u\|_{H_{\gamma}^{\varepsilon}}^{2} \leq c_{2}\left(1+\varepsilon^{\gamma-1}\right)\|u\|_{V_{1}^{\varepsilon} \times V_{2}^{\varepsilon}}^{2} \quad \forall u \in H_{\gamma}^{\varepsilon} .
$$


Let us now turn back to problem (2.2), whose variational formulation is

$$
\left\{\begin{array}{l}
\text { Find } u^{\varepsilon}=\left(u_{1}^{\varepsilon}, u_{2}^{\varepsilon}\right) \in H_{\gamma}^{\varepsilon} \text { such that } \\
\int_{\Omega_{1}^{\varepsilon}} A^{\varepsilon} \nabla u_{1}^{\varepsilon} \nabla v_{1} d x+\int_{\Omega_{2}^{\varepsilon}} A^{\varepsilon} \nabla u_{2}^{\varepsilon} \nabla v_{2} d x+\int_{\Omega_{1}^{\varepsilon}} v_{1} h_{1}^{\varepsilon}\left(x, u_{1}^{\varepsilon}\right) d x \\
+\int_{\Omega_{2}^{\varepsilon}} v_{2} h_{2}^{\varepsilon}\left(x, u_{2}^{\varepsilon}\right) d x+\varepsilon^{\gamma+1} \int_{\Gamma^{\varepsilon}}\left(v_{1}-v_{2}\right) h^{\varepsilon}\left(x, u_{1}^{\varepsilon}-u_{2}^{\varepsilon}\right) d \sigma \\
=\int_{\Omega_{1}^{\varepsilon}} f v_{1} d x+\int_{\Omega_{2}^{\varepsilon}} f v_{2} d x \forall\left(v_{1}, v_{2}\right) \in H_{\gamma}^{\varepsilon} .
\end{array}\right.
$$

We state below an existence and uniqueness result for this problem, which is a particular case of Theorem 3.1 proved in the next section.

Theorem 2.6. Let the function $h$ satisfy assumptions $\left(\mathcal{H}_{1}\right),\left(\mathcal{H}_{2}\right)$ and $h_{1}, h_{2}$ satisfy assumptions $\left(\mathcal{H}_{1}\right),\left(\mathcal{H}_{4}\right)$. Suppose further that $h$ or $h_{2}$ fulfills assumption $\left(\mathcal{H}_{3}\right)$. Then, for every fixed $\varepsilon$, problem (2.10) has a solution $u^{\varepsilon} \in H_{\gamma}^{\varepsilon}$.

If moreover $h$ or $h_{2}$ is strictly increasing, then the solution is unique.

We end this section by some a priori estimates for problem (2.10).

Proposition 2.7. (a priori estimates) Let $\gamma \leq 1$ and suppose that the function $h$ satisfies assumptions $\left(\mathcal{H}_{1}\right)-\left(\mathcal{H}_{3}\right)$ and $h_{1}, h_{2}$ satisfy assumptions $\left(\mathcal{H}_{1}\right),\left(\mathcal{H}_{4}\right)$. If $u^{\varepsilon}=\left(u_{1}^{\varepsilon}, u_{2}^{\varepsilon}\right)$ is a weak solution of problem (2.2), then there exists a positive constant $c$, independent of $\varepsilon$, such that

$$
\left\{\begin{array}{l}
\left\|\left(\nabla u_{1}^{\varepsilon}, \nabla u_{2}^{\varepsilon}\right)\right\|_{L^{2}\left(\Omega_{1}^{\varepsilon}\right) \times L^{2}\left(\Omega_{2}^{\varepsilon}\right)} \leq c, \\
\left\|u_{1}^{\varepsilon}-u_{2}^{\varepsilon}\right\|_{L^{2}\left(\Gamma^{\varepsilon}\right)} \leq c \varepsilon^{-\gamma / 2} .
\end{array}\right.
$$

Proof. With the assumptions given as above, problem (2.2) has a weak solution due to Theorem 2.6. Arguing as in the linear case [26], together with the coerciveness of $h$, the proof of (2.11) is straightforward.

Remark 2.8. For $\gamma=1$, if the coerciveness assumption on $h$ is replaced by that on $h_{2}$, then we still deduce the a priori estimates in (2.11) by using (2.9).

\section{An existence and uniqueness result}

Let $\mathcal{O}$ be a Lipschitz bounded open set of $\mathbb{R}^{n}$. We take $\mathcal{O}_{1}, \mathcal{O}_{2}$ as the open disjoint subsets of $\mathcal{O}$ such that $\mathcal{O}_{1}$ is connected and $\mathcal{O}_{2}$ has a finite number of connected components. We also assume that the interface $S$ between $\mathcal{O}_{1}, \mathcal{O}_{2}$ is Lipschitz continuous and set $S_{1}=\partial \mathcal{O} \cap \partial \mathcal{O}_{1}, S_{2}=\partial \mathcal{O} \cap \partial \mathcal{O}_{2}$.

The main result of this section concerns the existence and the uniqueness of a weak solution of the following more general problem:

$$
\begin{cases}-\operatorname{div}\left(B \nabla u_{1}\right)+h_{1}\left(x, u_{1}\right)=f & \text { in } \mathcal{O}_{1}, \\ -\operatorname{div}\left(B \nabla u_{2}\right)+h_{2}\left(x, u_{2}\right)=f & \text { in } \mathcal{O}_{2}, \\ B \nabla u_{1} \cdot n_{1}=-B \nabla u_{2} \cdot n_{2} & \text { on } S, \\ -B \nabla u_{1} \cdot n_{1}=h\left(x, \delta\left(u_{1}-u_{2}\right)\right) & \text { on } S, \\ u_{1}=0 & \text { on } S_{1}, \\ u_{2}=0 & \text { on } S_{2},\end{cases}
$$

for a given $\delta \in \mathbb{R}$. 
The existence and uniqueness result stated in Theorem 2.6 will follow as a particular case.

Let us define the functional space $H$ as follows

$$
H \doteq\left\{u=\left(u_{1}, u_{2}\right) \mid u_{1} \in V_{1}, u_{2} \in V_{2}\right\},
$$

endowed with the norm

$$
\|u\|_{H}^{2}=\left\|\nabla u_{1}\right\|_{L^{2}\left(\mathcal{O}_{1}\right)}^{2}+\left\|\nabla u_{2}\right\|_{L^{2}\left(\mathcal{O}_{2}\right)}^{2}+\left\|u_{1}-u_{2}\right\|_{L^{2}(S)}^{2} \quad \forall u=\left(u_{1}, u_{2}\right) \in H,
$$

where

$$
V_{i}=\left\{v \in H^{1}\left(\mathcal{O}_{i}\right): v=0 \text { on } S_{i}\right\}, \quad i=1,2,
$$

with the norms $\|v\|_{V_{1}}=\|\nabla v\|_{L^{2}\left(\mathcal{O}_{1}\right)}$ and $\|v\|_{V_{2}}=\|v\|_{H^{1}\left(\mathcal{O}_{2}\right)}$.

N.B. In this section, we suppose that all assumptions $\mathcal{H}$ introduced in Sect. 2 are written with $\mathcal{O}$ instead of $Y$ and without any periodicity assumptions.

Now we introduce an assumption more general than $\left(\mathcal{H}_{2}\right)$, which is enough to obtain the results for the existence and the uniqueness.

Assumption $\mathcal{H}_{2}^{\prime}$ : The function $g(y, s): Y \times \mathbb{R} \longrightarrow \mathbb{R}$ satisfies assumption $\left(\mathcal{H}_{2}^{\prime}\right)$ iff there exists a constant $c>0$ and an exponent $q$, with

$$
1 \leq q<+\infty \text { if } n=2 \text { and } 1 \leq q \leq \frac{n}{n-2} \text { if } n>2
$$

such that

$$
\left|\frac{\partial g}{\partial s}(y, s)\right| \leq c\left(1+|s|^{q-1}\right) \quad \forall(y, s) \in Y \times \mathbb{R} .
$$

Theorem 3.1. Let $f \in L^{2}(\mathcal{O})$ and $B \in M(\alpha, \beta, \mathcal{O})$. Suppose that the function $h$ satisfies assumptions $\left(\mathcal{H}_{1}\right),\left(\mathcal{H}_{2}^{\prime}\right)$ and $h_{1}, h_{2}$ satisfy assumptions $\left(\mathcal{H}_{1}\right),\left(\mathcal{H}_{4}\right)$. Assume further that $h$ or $h_{2}$ satisfies assumption $\left(\mathcal{H}_{3}\right)$. Then, there exists a solution $u=\left(u_{1}, u_{2}\right) \in H$ of the following variational formulation of problem (3.1):

$$
\left\{\begin{array}{l}
\text { Find } u=\left(u_{1}, u_{2}\right) \in H \text { such that } \\
\int_{\mathcal{O}_{1}} B \nabla u_{1} \nabla v_{1} d x+\int_{\mathcal{O}_{2}} B \nabla u_{2} \nabla v_{2} d x+\int_{\mathcal{O}_{1}} v_{1} h_{1}\left(x, u_{1}\right) d x \\
\quad+\int_{\mathcal{O}_{2}} v_{2} h_{2}\left(x, u_{2}\right) d x+\int_{S}\left(v_{1}-v_{2}\right) h\left(x, \delta\left(u_{1}-u_{2}\right)\right) d \sigma \\
=\int_{\mathcal{O}_{1}} f v_{1} d x+\int_{\mathcal{O}_{2}} f v_{2} d x \quad \forall\left(v_{1}, v_{2}\right) \in H .
\end{array}\right.
$$

Furthermore, the solution is unique if $h$ or $h_{2}$ is strictly increasing.

This theorem is proved at the end of this section, after some preliminary results. The next proposition shows in particular that all the terms in (3.2) make sense:

Proposition 3.2. Let the function $h$ satisfy assumptions $\left(\mathcal{H}_{1}\right),\left(\mathcal{H}_{2}^{\prime}\right)$ and the functions $h_{1}, h_{2}$ satisfy $\left(\mathcal{H}_{1}\right),\left(\mathcal{H}_{4}\right)$. Suppose that $r, r^{\prime}, s$, s' are such that

(i) if $n=2, r, s \in\left[1,+\infty\left[\right.\right.$ and $r \geq q r^{\prime}, s \geq p s^{\prime}$, 
(ii) if $n>2, r=\frac{2(n-1)}{n-2}, s=2^{*}=\frac{2 n}{n-2}$ and $\frac{1}{r}+\frac{1}{r^{\prime}}=\frac{1}{s}+\frac{1}{s^{\prime}}=1$.

Then, the following three maps are bounded and continuous:

$$
\begin{aligned}
J: u=\left(u_{1}, u_{2}\right) & \in H \longmapsto J(u) \in L^{r^{\prime}}(S), \\
J_{i}: u_{i} & \in H^{1}\left(\mathcal{O}_{i}\right) \longmapsto J_{i}\left(u_{i}\right) \in L^{s^{\prime}}\left(\mathcal{O}_{i}\right), \quad i=1,2,
\end{aligned}
$$

where $J(u)(x)=h\left(x, \delta\left(u_{1}(x)-u_{2}(x)\right)\right), J_{i}\left(u_{i}\right)(x)=h_{i}\left(x, u_{i}(x)\right)$ and for every $u_{i}, v_{i} \in H^{1}\left(\mathcal{O}_{i}\right), i=1,2$,

$$
\left\{\begin{array}{l}
\left(v_{1}-v_{2}\right) h\left(x, \delta\left(u_{1}-u_{2}\right)\right) \in L^{1}(S), \\
v_{i} h_{i}\left(x, u_{i}\right) \in L^{1}\left(\mathcal{O}_{i}\right) .
\end{array}\right.
$$

Moreover, there exists a positive constant $c$ such that

$$
\|J(u)\|_{L^{r^{\prime}(S)}} \leq c\left(1+\left\|u_{1}-u_{2}\right\|_{L^{r}(S)}^{r / r^{\prime}}\right) .
$$

Proof. Let us first note that $J=g_{1} \circ g_{2}$ where

$$
g_{1}: v \in L^{r}(S) \longmapsto g_{1}(v) \in L^{r^{\prime}}(S)
$$

is defined by $g_{1}(v)(x)=h(x, v(x))$ and

$$
g_{2}: u=\left(u_{1}, u_{2}\right) \in H \longmapsto u_{1}-u_{2} \in L^{r}(S) .
$$

Taking into account that $h$ satisfies $\left(\mathcal{H}_{1}\right)$ and $\left(\mathcal{H}_{2}^{\prime}\right)$, by the classical result in [32], $g_{1}$ is continuous and bounded. On the other hand, arguing as in $[7,16]$, the linear continuity of the inclusions $H^{1}\left(\mathcal{O}_{i}\right) \subset L^{r}(S)$ for $i=1,2$, gives that $g_{2}$ is bounded and continuous. Hence, the operator $J$ is bounded and continuous from $H$ into $L^{r^{\prime}}(S)$, which provides $(3.3)_{1}$.

We now turn our attention to the boundedness and the continuity of the two maps $J_{1}, J_{2}$. If $n>2$, the embeddings $H^{1}\left(\mathcal{O}_{i}\right) \subset L^{2^{*}}\left(\mathcal{O}_{i}\right)$ for $i=1,2$, are continuous.

Then, taking $v_{i} \in H^{1}\left(\mathcal{O}_{i}\right)$, since the functions $h_{1}, h_{2}$ satisfy $\left(\mathcal{H}_{4}\right)$ and $p_{1} \leq \frac{n+2}{n-2}=\frac{2^{*}}{\left(2^{*}\right)^{\prime}}$, we have

$$
\left|h_{i}\left(x, v_{i}\right)\right| \leq c\left(1+\left|v_{i}\right|^{p_{1}}\right) \leq 2 c\left(1+\left|v_{i}\right|^{\frac{2^{*}}{\left(2^{*}\right)^{\prime}}}\right), \quad \text { for } i=1,2 .
$$

Consequently, the functions $J_{i}, i=1,2$, are bounded and continuous from $H^{1}\left(\mathcal{O}_{i}\right)$ into $L^{\left(2^{*}\right)^{\prime}}\left(\mathcal{O}_{i}\right)$, which implies $(3.3)_{2}$.

For $n=2$ we argue in a similar way as above, using the continuous embedding $H^{1}\left(\mathcal{O}_{i}\right) \subset L^{s}\left(\mathcal{O}_{i}\right), i=1,2, \forall s \in[1,+\infty[$.

Finally, inequality (3.4) comes from similar arguments as in [7].

Let us now recall a result from [36] that we shall need in the sequel.

Lemma 3.3. [36] There exists a constant $c>0$ such that

$$
\|u\|_{L^{2}\left(\mathcal{O}_{1}\right) \times L^{2}\left(\mathcal{O}_{2}\right)} \leq c\|u\|_{H},
$$

for every $u \in H$. 
Proof of Theorem 3.1. We first prove the existence of the solution by using the Minty-Browder theorem for the operator $G: u=\left(u_{1}, u_{2}\right) \in H \mapsto G(u) \in H^{\prime}$ where

$$
\begin{aligned}
\langle G(u), v\rangle_{H^{\prime}, H}= & \sum_{i=1,2} \int_{\mathcal{O}_{i}} B \nabla u_{i} \nabla v_{i} d x+\sum_{i=1,2} \int_{\mathcal{O}_{i}} v_{i} h_{i}\left(x, u_{i}\right) d x \\
& +\int_{S}\left(v_{1}-v_{2}\right) h\left(x, \delta\left(u_{1}-u_{2}\right)\right) d \sigma-\sum_{i=1,2} \int_{\mathcal{O}_{i}} f v_{i} d x .
\end{aligned}
$$

Clearly, the continuity and the boundedness of $G$ follow from Proposition 3.2. Moreover, the fact that the functions $h, h_{1}$ and $h_{2}$ satisfy assumption $\left(\mathcal{H}_{1}\right)$ provides the monotonicity of $G$.

Let us turn our attention into the coerciveness of $G$. If $h$ satisfies assumption $\left(\mathcal{H}_{3}\right)$, taking into account Remark 2.2 and Lemma 3.3, we have

$$
\begin{aligned}
\langle G(u), u\rangle_{H^{\prime}, H} \geq & \alpha\left\|\nabla u_{1}\right\|_{L^{2}\left(\mathcal{O}_{1}\right)}^{2}+\alpha\left\|\nabla u_{2}\right\|_{L^{2}\left(\mathcal{O}_{2}\right)}^{2}+c\left\|u_{1}-u_{2}\right\|_{L^{2}(S)}^{2} \\
& -\|f\|_{L^{2}(\mathcal{O})}\left(\left\|u_{1}\right\|_{L^{2}\left(\mathcal{O}_{1}\right)}+\left\|u_{2}\right\|_{L^{2}\left(\mathcal{O}_{2}\right)}\right) \\
\geq & c_{1}\|u\|_{H}^{2}-c_{2}\|u\|_{H}, \quad \forall u \in H,
\end{aligned}
$$

If the coerciveness assumption of $h$ is replaced by that of $h_{2}$, then

$$
\begin{aligned}
\langle G(u), u\rangle_{H^{\prime}, H} \geq & \alpha\left\|\nabla u_{1}\right\|_{L^{2}\left(\mathcal{O}_{1}\right)}^{2}+\alpha\left\|\nabla u_{2}\right\|_{L^{2}\left(\mathcal{O}_{2}\right)}^{2}+c\left\|u_{2}\right\|_{L^{2}\left(\mathcal{O}_{2}\right)}^{2} \\
& -\|f\|_{L^{2}(\mathcal{O})}\left(\left\|u_{1}\right\|_{L^{2}\left(\mathcal{O}_{1}\right)}+\left\|u_{2}\right\|_{L^{2}\left(\mathcal{O}_{2}\right)}\right) \\
\geq & c\left(\left\|u_{1}\right\|_{H^{1}\left(\mathcal{O}_{1}\right)}^{2}+\left\|u_{2}\right\|_{H^{1}\left(\mathcal{O}_{2}\right)}^{2}\right)-c_{1}\|u\|_{H} \\
\geq & c_{2}\|u\|_{H}^{2}-c_{1}\|u\|_{H}, \quad \forall u \in H
\end{aligned}
$$

Obviously, the above inequalities imply that $G$ is coercive.

Hence, by the Minty-Browder theorem, the operator $G$ is surjective so that there exists $u \in H$ such that $G(u)=0$, that is a solution $u \in H$ of problem (3.2).

Let us now check the uniqueness of the solution.

Suppose that problem (3.1) has two solutions $u=\left(u_{1}, u_{2}\right), w=$ $\left(w_{1}, w_{2}\right) \in H$. Choosing as test function $v=u-w$ in (3.2), we have

$$
\begin{aligned}
& \sum_{i=1,2} \int_{\mathcal{O}_{i}} B\left(\nabla u_{i}-\nabla w_{i}\right)\left(\nabla u_{i}-\nabla w_{i}\right) d x \\
& \quad+\sum_{i=1,2} \int_{\mathcal{O}_{i}}\left(u_{i}-w_{i}\right)\left[h_{i}\left(x, u_{i}\right)-h_{i}\left(x, w_{i}\right)\right] d x \\
& \quad+\int_{S}\left[h\left(x, \delta\left(u_{1}-u_{2}\right)\right)-h\left(x, \delta\left(w_{1}-w_{2}\right)\right)\right]\left[\left(u_{1}-u_{2}\right)-\left(w_{1}-w_{2}\right)\right] d \sigma=0 .
\end{aligned}
$$


By virtue of the ellipticity of $B$, the monotonicity of $h, h_{1}, h_{2}$ and the fact that $h\left(\right.$ or $\left.h_{2}\right)$ is strictly increasing, equality (3.5) gives

$$
\begin{cases}\nabla\left(u_{i}-w_{i}\right)=0 & \text { in } \mathcal{O}_{i} \quad \text { for } i=1,2 \\ u_{1}-u_{2}=w_{1}-w_{2} & \text { on } S\left(\text { or } u_{2}=w_{2} \text { in } \mathcal{O}_{2}\right) .\end{cases}
$$

Then, using the Poincaré inequality in $V_{1}$ we obtain $u \equiv w$, which ends the proof.

\section{Periodic unfolding method for two-component domains}

In this section, we first recall the unfolding operators $\mathcal{T}_{1}^{\varepsilon}, \mathcal{T}_{2}^{\varepsilon}$ for the twocomponent domain and the boundary unfolding operator $\mathcal{T}_{b}^{\varepsilon}$ together with their main properties.

The operators $\mathcal{T}_{1}^{\varepsilon}$ and $\mathcal{T}_{b}^{\varepsilon}$ have been introduced in $[13,15,26]$ and $\mathcal{T}_{2}^{\varepsilon}$ in [26], where the relationship between the traces of $\mathcal{T}_{1}^{\varepsilon}$ and $\mathcal{T}_{2}^{\varepsilon}$ is also studied. Although as mentioned in Section 2 the domain $\Omega_{2}^{\varepsilon}$ defined in [26] is somehow different, all the results therein concerning the unfolding operators are still true for this case. Indeed, the main difference concerns $\mathcal{T}_{1}^{\varepsilon}$, due to the fact that $u_{1}^{\varepsilon}$ does not belong to $V^{\varepsilon} \doteq\left\{v \in H^{1}\left(\Omega_{1}^{\varepsilon}\right) \mid v=0\right.$ on $\left.\partial \Omega\right\}$ as in the linear case studied in [26], but belongs to $V_{1}^{\varepsilon}$ (see Definition 2.3). Also, the fact that the domain $\Omega_{2}^{\varepsilon}$ contains some more holes (or part of them) does not make any relevant difference. Consequently, it is easy to check, taking into account [13, Theorem 2.13], that the convergence results recalled in Theorem 4.3 below are still true in this case.

In the second part of this section, we state some results concerning the composed operators $\mathcal{T}_{b}^{\varepsilon} \circ h$ and $\mathcal{T}_{i}^{\varepsilon} \circ h_{i}, i=1,2$ where $h, h_{1}$ and $h_{2}$ are the nonlinear functions in problem (2.2). Moreover, we prove (see Theorem 4.5 below) a new strong convergence result for $\mathcal{T}_{2}^{\varepsilon}\left(u_{2}^{\varepsilon}\right)$, which is needed to pass to the limit in the unfolded nonlinear term concerning $h_{2}$ in the variational formulation (2.10).

In order to define the periodic unfolding operators, let us introduce the following sets as in [13] (see Fig. 2).

- $\widehat{K}_{\varepsilon}=\left\{k \in \mathbb{Z}^{n} \mid \varepsilon Y^{k} \subset \Omega\right\}, \widehat{\Omega}_{\varepsilon}=i n t \bigcup_{k \in \widehat{K}_{\varepsilon}} \varepsilon\left(k_{l}+\bar{Y}\right), \Lambda_{\varepsilon}=\Omega \backslash \widehat{\Omega}_{\varepsilon}$,

- $\widehat{\Omega}_{i}^{\varepsilon}=\bigcup_{k \in \widehat{K}_{\varepsilon}} \varepsilon Y_{i}^{k}, \quad \Lambda_{i}^{\varepsilon}=\Omega_{i}^{\varepsilon} \backslash \widehat{\Omega}_{i}^{\varepsilon}, \quad i=1,2$.

\subsection{The periodic unfolding operators}

Definition 4.1. For any function $\phi$ Lebesgue-measurable on $\Omega_{i}^{\varepsilon}$, the periodic unfolding operators $\mathcal{T}_{i}^{\varepsilon}, i=1,2$ are defined by the formula

$$
\mathcal{T}_{i}^{\varepsilon}(\phi)(x, y)= \begin{cases}\phi\left(\varepsilon\left[\frac{x}{\varepsilon}\right]_{Y}+\varepsilon y\right) & \text { a.e. }(x, y) \in \widehat{\Omega}_{\varepsilon} \times Y_{i}, \\ 0 & \text { a.e. }(x, y) \in \Lambda_{\varepsilon} \times Y_{i} .\end{cases}
$$




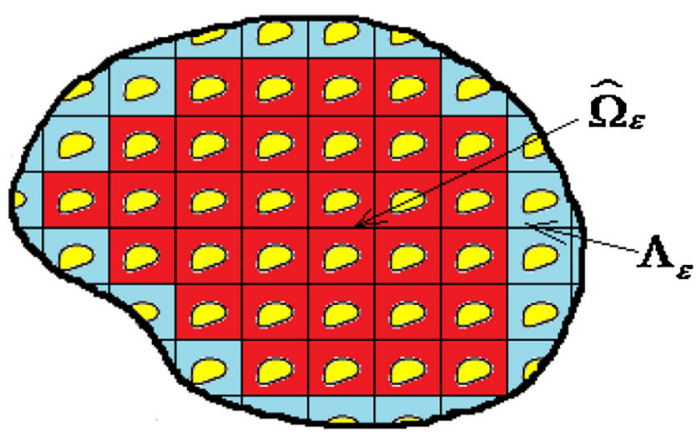

Figure 2. The sets $\widehat{\Omega}_{\varepsilon}$ and $\Lambda_{\varepsilon}$

For any function $\phi$ Lebesgue-measurable on $\Gamma^{\varepsilon}$, the periodic boundary unfolding operator $\mathcal{T}_{b}^{\varepsilon}$ is defined by

$$
\mathcal{T}_{b}^{\varepsilon}(\phi)(x, y)= \begin{cases}\phi\left(\varepsilon\left[\frac{x}{\varepsilon}\right]_{Y}+\varepsilon y\right) & \text { a.e. }(x, y) \in \widehat{\Omega}_{\varepsilon} \times \Gamma, \\ 0 & \text { a.e. }(x, y) \in \Lambda_{\varepsilon} \times \Gamma .\end{cases}
$$

For the readers' convenience, we recall the main properties of $\mathcal{T}_{i}^{\varepsilon}$ for $i=1,2$ as follows:

Proposition 4.2. $[13,15,26]$ For $p \in\left[1,+\infty\left[\right.\right.$, the operators $\mathcal{T}_{i}^{\varepsilon}$ are linear and continuous from $L^{p}\left(\Omega_{i}^{\varepsilon}\right)$ to $L^{p}(\Omega \times Y), i=1,2$; moreover,

(i) $\mathcal{T}_{i}^{\varepsilon}(\varphi \psi)=\mathcal{T}_{i}^{\varepsilon}(\varphi) \mathcal{T}_{i}^{\varepsilon}(\psi)$, for every $\varphi$, $\psi$ Lebesgue-measurable on $\Omega_{i}^{\varepsilon}$.

(ii) For every $\varphi \in L^{1}\left(\Omega_{i}^{\varepsilon}\right)$, one has

$\frac{1}{|Y|} \int_{\Omega \times Y_{i}} \mathcal{T}_{i}^{\varepsilon}(\varphi)(x, y) d x d y=\int_{\widehat{\Omega}_{i}^{\varepsilon}} \varphi(x) d x=\int_{\Omega_{i}^{\varepsilon}} \varphi(x) d x-\int_{\Lambda_{i}^{\varepsilon}} \varphi(x) d x$.

(iii) For every $\varphi \in L^{p}\left(\Omega_{i}^{\varepsilon}\right)$,

$$
\left\|\mathcal{T}_{i}^{\varepsilon}(\varphi)\right\|_{L^{p}\left(\Omega \times Y_{i}\right)} \leq|Y|^{1 / p}\|\varphi\|_{L^{p}\left(\Omega_{i}^{\varepsilon}\right)} .
$$

(iv) For $\varphi \in L^{p}(\Omega)$,

$$
\mathcal{T}_{i}^{\varepsilon}(\varphi) \longrightarrow \varphi \text { strongly in } L^{p}\left(\Omega \times Y_{i}\right) .
$$

(v) Let $\left\{\varphi_{\varepsilon}\right\}$ be a sequence in $L^{p}(\Omega)$ such that $\varphi_{\varepsilon} \longrightarrow \varphi$ strongly in $L^{p}(\Omega)$. Then,

$$
\mathcal{T}_{i}^{\varepsilon}\left(\varphi^{\varepsilon}\right) \longrightarrow \varphi \text { strongly in } L^{p}\left(\Omega \times Y_{i}\right)
$$

(vi) Let $\varphi \in L^{p}\left(Y_{i}\right)$ be a $Y$-periodic function and set $\varphi^{\varepsilon}(x)=\varphi(x / \varepsilon)$. Then $\mathcal{T}_{i}^{\varepsilon}\left(\varphi^{\varepsilon}\right) \longrightarrow \varphi$ strongly in $L^{p}\left(\Omega \times Y_{i}\right)$.

(vii) Let $\varphi_{\varepsilon} \in L^{p}\left(\Omega_{i}^{\varepsilon}\right)$ satisfy $\left\|\varphi_{\varepsilon}\right\|_{L^{p}\left(\Omega_{i}^{\varepsilon}\right)} \leq C$. If $\mathcal{T}_{i}^{\varepsilon}\left(\varphi_{\varepsilon}\right) \rightarrow \widehat{\varphi}$ weakly in $L^{p}\left(\Omega \times Y_{i}\right)$, then

$$
\widetilde{\varphi}_{\varepsilon} \rightarrow \theta_{i} \mathcal{M}_{Y_{i}}(\widehat{\varphi}) \quad \text { weakly in } L^{p}(\Omega) \text {. }
$$

(viii) If $\varphi \in W^{1, p}\left(\Omega_{i}^{\varepsilon}\right)$, then $\nabla_{y}\left[\mathcal{T}_{i}^{\varepsilon}(\varphi)\right]=\varepsilon \mathcal{T}_{i}^{\varepsilon}(\nabla \varphi)$ and $\mathcal{T}_{i}^{\varepsilon}(\varphi) \in$ $L^{p}\left(\Omega, W^{1, p}\left(Y_{i}\right)\right)$. 
(ix) If $\varphi \in L^{s}\left(\Gamma^{\varepsilon}\right)$ for $s \in[1,+\infty[$, then

$$
\left\|\mathcal{T}_{b}^{\varepsilon}(\varphi)\right\|_{L^{s}(\Omega \times \Gamma)} \leq|Y|^{1 / s} \varepsilon^{1 / s}\|\varphi\|_{L^{s}\left(\Gamma^{\varepsilon}\right)} .
$$

Let us now recall the main convergence results which have been proved in $[13,26]$.

Theorem 4.3. $[13,26]$ Let $\gamma \in \mathbb{R}$ and $u^{\varepsilon}=\left(u_{1}^{\varepsilon}, u_{2}^{\varepsilon}\right)$ be a bounded sequence in $H_{\gamma}^{\varepsilon}$. Then,

$$
\left\|\mathcal{T}_{1}^{\varepsilon}\left(u_{1}^{\varepsilon}\right)-\mathcal{T}_{2}^{\varepsilon}\left(u_{2}^{\varepsilon}\right)\right\|_{L^{2}(\Omega \times \Gamma)} \leq C \varepsilon^{\frac{1-\gamma}{2}},
$$

and there exists a subsequence (still denoted $\varepsilon$ ), $u_{1} \in H_{0}^{1}(\Omega)$ and $\widehat{u}_{1} \in$ $L^{2}\left(\Omega, H_{\text {per }}^{1}\left(Y_{1}\right)\right)$ with $\mathcal{M}_{\Gamma}\left(\widehat{u}_{1}\right)=0$ a.e. $x \in \Omega$ such that

$$
\begin{cases}\mathcal{T}_{1}^{\varepsilon}\left(u_{1}^{\varepsilon}\right) \longrightarrow u_{1} & \text { strongly in } L^{2}\left(\Omega, H^{1}\left(Y_{1}\right)\right), \\ \mathcal{T}_{1}^{\varepsilon}\left(\nabla u_{1}^{\varepsilon}\right) \rightarrow \nabla u_{1}+\nabla_{y} \widehat{u}_{1} & \text { weakly in } L^{2}\left(\Omega \times Y_{1}\right) .\end{cases}
$$

Moreover, if $\gamma \leq 1$, there exists a subsequence (still denoted $\varepsilon$ ), $u_{2} \in$ $L^{2}(\Omega)$ and $\bar{u}_{2} \in L^{2}\left(\Omega, H^{1}\left(Y_{2}\right)\right)$ with $\mathcal{M}_{\Gamma}\left(\bar{u}_{2}\right)=0$ a.e. $x \in \Omega$ such that

$$
\begin{cases}\mathcal{T}_{2}^{\varepsilon}\left(u_{2}^{\varepsilon}\right) \rightarrow u_{2} & \text { weakly in } L^{2}\left(\Omega, H^{1}\left(Y_{2}\right)\right) \\ \mathcal{T}_{2}^{\varepsilon}\left(\nabla u_{2}^{\varepsilon}\right) \rightarrow \nabla_{y} \bar{u}_{2} & \text { weakly in } L^{2}\left(\Omega \times Y_{2}\right)\end{cases}
$$

Furthermore, if $\gamma<1$, then $u_{1}=u_{2}$ and

(i) if $\gamma<-1$, we have

$$
\widehat{u}_{1}=\bar{u}_{2}-y_{\Gamma} \nabla u_{1} \quad \text { on } \Omega \times \Gamma,
$$

where $y_{\Gamma}=y-\mathcal{M}_{\Gamma}(y)$.

(ii) if $\gamma=-1$, there exists $\xi_{\Gamma} \in L^{2}(\Omega)$ such that

$$
\frac{\mathcal{T}_{1}^{\varepsilon}\left(u_{1}^{\varepsilon}\right)-\mathcal{T}_{2}^{\varepsilon}\left(u_{2}^{\varepsilon}\right)}{\varepsilon} \rightarrow \widehat{u}_{1}-\bar{u}_{2}+y_{\Gamma} \nabla u_{1}+\xi_{\Gamma} \quad \text { weakly in } L^{2}(\Omega \times \Gamma) .
$$

Remark 4.4. From now on, we set

$$
\widehat{u}_{2}=\bar{u}_{2}-y_{\Gamma} \nabla u_{1}-\xi_{\Gamma} \in L^{2}\left(\Omega, H^{1}\left(Y_{2}\right)\right) .
$$

With this notation, in (4.2) 2 we can replace $\nabla_{y} \bar{u}_{2}$ by $\nabla u_{1}+\nabla_{y} \widehat{u}_{2}$ and (4.3)(4.4) can be rewritten respectively as follows:

$$
\begin{gathered}
\widehat{u}_{1}=\widehat{u}_{2}+\xi_{\Gamma} \quad \text { on } \Omega \times \Gamma \quad \text { if } \gamma<-1, \\
\mathcal{T}_{1}^{\varepsilon}\left(u_{1}^{\varepsilon}\right)-\mathcal{T}_{2}^{\varepsilon}\left(u_{2}^{\varepsilon}\right) / \varepsilon \rightarrow \widehat{u}_{1}-\widehat{u}_{2} \quad \text { weakly in } L^{2}(\Omega \times \Gamma) \quad \text { if } \gamma=-1 .
\end{gathered}
$$

Let us mention that, when comparing to [26], the notations of $\widehat{u}_{2}$ and $\bar{u}_{2}$ used here are interchanged, in order to simplify the presentation. 


\subsection{Some convergence results}

The main difficulty when passing to the limit in the unfolded version of problem (2.2) is clearly given by the nonlinear terms. Concerning $\mathcal{T}_{2}^{\varepsilon}\left(u_{2}^{\varepsilon}\right)$, its weak convergence in $L^{2}\left(\Omega, H^{1}\left(Y_{2}\right)\right)$ provided by Theorem 4.3 is not sufficient.

This is why we first need to prove that the above convergence is actually strong. We do that in the next theorem, then we obtain the convergences of the unfolded nonlinear terms.

Theorem 4.5. Let $\gamma<1$ and $u^{\varepsilon}=\left(u_{1}^{\varepsilon}, u_{2}^{\varepsilon}\right)$ be a bounded sequence in $H_{\gamma}^{\varepsilon}$. Then, there exists a subsequence (still denoted $\varepsilon$ ) and $u_{1} \in H_{0}^{1}(\Omega)$ such that

$$
\begin{cases}\mathcal{T}_{2}^{\varepsilon}\left(u_{2}^{\varepsilon}\right) \longrightarrow u_{1} & \text { strongly in } L^{2}\left(\Omega, H^{1}\left(Y_{2}\right)\right), \\ \mathcal{T}_{1}^{\varepsilon}\left(u_{1}^{\varepsilon}\right)-\mathcal{T}_{2}^{\varepsilon}\left(u_{2}^{\varepsilon}\right) \longrightarrow 0 & \text { strongly in } L^{2}\left(\Omega, L^{r}(\Gamma)\right),\end{cases}
$$

where $r$ is defined in Proposition 3.2.

Proof. From the proof of Theorem 2.18 in [26], we have

$$
\mathcal{T}_{2}^{\varepsilon}\left(u_{2}^{\varepsilon}\right) \longrightarrow u_{1} \quad \text { strongly in } L^{2}(\Omega \times \Gamma) \text {. }
$$

Moreover, since $Y_{2}$ is bounded with a Lipschitz boundary $\Gamma$, by the Friedrichs inequality we have for some positive constant $c$,

$$
\|v\|_{L^{2}\left(Y_{2}\right)}^{2} \leq c\left(\|v\|_{L^{2}(\Gamma)}^{2}+\|\nabla v\|_{L^{2}\left(Y_{2}\right)}^{2}\right) \quad \forall v \in H^{1}\left(Y_{2}\right)
$$

which applied to the function $\mathcal{T}_{2}^{\varepsilon}\left(u_{2}^{\varepsilon}\right)-u_{1}$ gives

$$
\left\|\mathcal{T}_{2}^{\varepsilon}\left(u_{2}^{\varepsilon}\right)-u_{1}\right\|_{L^{2}\left(\Omega \times Y_{2}\right)}^{2} \leq c\left(\left\|\mathcal{T}_{2}^{\varepsilon}\left(u_{2}^{\varepsilon}\right)-u_{1}\right\|_{L^{2}(\Omega \times \Gamma)}^{2}+\left\|\nabla_{y} \mathcal{T}_{2}^{\varepsilon}\left(u_{2}^{\varepsilon}\right)\right\|_{L^{2}\left(\Omega \times Y_{2}\right)}^{2}\right),
$$

since $u_{1}$ is independent of $y$. Note that

$$
\left\|\nabla_{y} \mathcal{T}_{2}^{\varepsilon}\left(u_{2}^{\varepsilon}\right)\right\|_{L^{2}\left(\Omega \times Y_{2}\right)}=\varepsilon\left\|\mathcal{T}_{2}^{\varepsilon}\left(\nabla u_{2}^{\varepsilon}\right)\right\|_{L^{2}\left(\Omega \times Y_{2}\right)} \leq c \varepsilon,
$$

in view of Proposition 4.2(iii), (viii) and the fact that $u^{\varepsilon}$ is a bounded sequence in $H_{\gamma}^{\varepsilon}$. Taking into account (4.9)-(4.11), we deduce $(4.8)_{1}$.

For the second convergence in (4.8) one has

$$
\begin{aligned}
& \int_{\Omega}\left\|\mathcal{T}_{1}^{\varepsilon}\left(u_{1}^{\varepsilon}\right)-\mathcal{T}_{2}^{\varepsilon}\left(u_{2}^{\varepsilon}\right)\right\|_{L^{r}(\Gamma)}^{2} d x \\
& \quad \leq 2 \int_{\Omega}\left\|\mathcal{T}_{1}^{\varepsilon}\left(u_{1}^{\varepsilon}\right)-u_{1}\right\|_{L^{r}(\Gamma)}^{2} d x+2 \int_{\Omega}\left\|\mathcal{T}_{2}^{\varepsilon}\left(u_{2}^{\varepsilon}\right)-u_{1}\right\|_{L^{r}(\Gamma)}^{2} d x \\
& \quad \leq c\left(\int_{\Omega}\left\|\mathcal{T}_{1}^{\varepsilon}\left(u_{1}^{\varepsilon}\right)-u_{1}\right\|_{H^{1}\left(Y_{1}\right)}^{2} d x+\int_{\Omega}\left\|\mathcal{T}_{2}^{\varepsilon}\left(u_{2}^{\varepsilon}\right)-u_{1}\right\|_{H^{1}\left(Y_{2}\right)}^{2} d x\right) \longrightarrow 0
\end{aligned}
$$

where we use $(4.1)_{1},(4.8)_{1}$ and the continuous embeddings $H^{1}\left(Y_{i}\right) \subset L^{r}(\Gamma)$, $i=1,2$ (see Proposition 3.2). This completes the proof.

The following lemma is needed to prove the next proposition.

Lemma 4.6. There exists a positive constant $c$, independent of $\varepsilon$, such that, for any $v \in V_{1}^{\varepsilon}$,

$$
\|v\|_{L^{s}\left(\Omega_{1}^{\varepsilon}\right)} \leq c\|v\|_{V_{1}^{\varepsilon}},
$$


for every $s \in\left[1,2^{*}\right]$ if $n>2$ and $s \in[1,+\infty[$ if $n=2$.

Proof. Let $\Omega_{0}$ be an open subset of $\mathbb{R}^{n}$ such that $\Omega \subset \Omega_{0}$ and $d\left(\partial \Omega_{0}, \Omega\right)>$ $\operatorname{diam}(Y)$. Set $\Omega_{0}^{\varepsilon}=\Omega_{0} \backslash \bigcup_{k \in \mathbb{Z}^{n}} \varepsilon \overline{Y_{2}^{k}}$. Observe that, for $\varepsilon$ small enough, $\partial \Omega_{0}$ does not meet the boundary of the holes intersecting $\partial \Omega$. Consequently, if $\widetilde{v}$ is the zero extension to $\Omega_{0}^{\varepsilon}$ of a function $v \in V_{1}^{\varepsilon}$, then according to Remark $2.4(\mathrm{i}), \widetilde{v} \in H^{1}\left(\Omega_{0}^{\varepsilon}\right)$ and $\widetilde{v}=0$ on $\partial \Omega_{0}^{\varepsilon}$.

Hence, from classical results in $[17,18]$, there exists an extension operator $P^{\varepsilon}$ from $H^{1}\left(\Omega_{0}^{\varepsilon}\right)$ to $H_{0}^{1}\left(\Omega_{0}\right)$ such that,

$$
\left\|P^{\varepsilon} \tilde{v}\right\|_{H_{0}^{1}\left(\Omega_{0}\right)} \leq c\|\tilde{v}\|_{H^{1}\left(\Omega_{0}^{\varepsilon}\right)},
$$

where the constant $c$ is independent of $\varepsilon$.

As a result, by using the Sobolev embedding $H_{0}^{1}\left(\Omega_{0}\right) \subset L^{s}\left(\Omega_{0}\right)$, we obtain

$$
\|v\|_{L^{s}\left(\Omega_{1}^{\varepsilon}\right)}=\|\widetilde{v}\|_{L^{s}\left(\Omega_{0}^{\varepsilon}\right)} \leq\left\|P^{\varepsilon} \widetilde{v}\right\|_{L^{s}\left(\Omega_{0}\right)} \leq c_{1}\left\|P^{\varepsilon} \widetilde{v}\right\|_{H_{0}^{1}\left(\Omega_{0}\right)} \leq c_{2}\|v\|_{V_{1}^{\varepsilon}},
$$

where the constant $c_{2}$ does not depend on $\varepsilon$, which concludes the proof.

Proposition 4.7. Let the function $h_{1}$ satisfy assumptions $\left(\mathcal{H}_{1}\right),\left(\mathcal{H}_{4}\right)$ and $h_{2}$ satisfy assumptions $\left(\mathcal{H}_{1}\right),\left(\mathcal{H}_{4}^{\prime}\right)$. If $u^{\varepsilon}=\left(u_{1}^{\varepsilon}, u_{2}^{\varepsilon}\right)$ is a bounded sequence in $H_{\gamma}^{\varepsilon}$, then there exists a subsequence (still denoted $\varepsilon$ ) and $u_{1} \in H_{0}^{1}(\Omega)$ such that

(i) $\mathcal{T}_{1}^{\varepsilon}\left(h_{1}^{\varepsilon}\left(x, u_{1}^{\varepsilon}\right)\right) \longrightarrow h_{1}\left(y, u_{1}\right)$ strongly in $L^{t / p_{1}}\left(\Omega \times Y_{1}\right)$,

(ii) $\mathcal{T}_{2}^{\varepsilon}\left(h_{2}^{\varepsilon}\left(x, u_{2}^{\varepsilon}\right)\right) \longrightarrow h_{2}\left(y, u_{1}\right)$ strongly in $L^{2 / p_{2}}\left(\Omega \times Y_{2}\right) \quad$ if $\gamma<1$, where

$$
\begin{cases}t=\max \left\{2, p_{1}\right\} & \text { if } n=2, \\
t \in\left[\max \left\{2, \frac{n+2}{n-2}\right\}, 2^{*}\left[\begin{array}{l}
\text { if } n>2 .
\end{array}\right.\right.\end{cases}
$$

Remark 4.8. In order to treat the nonlinear term by unfolding, in the sequel we use the fact that, from the definition of the unfolding operators and assumption $\left(\mathcal{H}_{1}\right)$ on $h, h_{1}$ and $h_{2}$,

$$
\left\{\begin{array}{l}
\mathcal{T}_{i}^{\varepsilon}\left(h_{i}^{\varepsilon}(\cdot, v)\right)(x, y)=h_{i}\left(y, \mathcal{T}_{i}^{\varepsilon}(v)(x, y)\right), \quad \text { for } i=1,2, \\
\mathcal{T}_{b}^{\varepsilon}\left(h^{\varepsilon}(\cdot, w)\right)(x, y)=h\left(y, \mathcal{T}_{b}^{\varepsilon}(w)(x, y)\right),
\end{array}\right.
$$

for any function $v$ measurable on $\Omega_{i}^{\varepsilon}$ and $w$ measurable on $\Gamma^{\epsilon}$.

Proof of Proposition 4.7. From Proposition 4.2(iii) and Lemma 4.6, one has

$$
\left\|\mathcal{T}_{1}^{\varepsilon}\left(u_{1}^{\varepsilon}\right)\right\|_{L^{s}\left(\Omega \times Y_{1}\right)} \leq|Y|^{1 / s}\left\|u_{1}^{\varepsilon}\right\|_{L^{s}\left(\Omega_{1}^{\varepsilon}\right)} \leq c\left\|\nabla u_{1}^{\varepsilon}\right\|_{L^{2}\left(\Omega_{1}^{\varepsilon}\right)} \leq c_{1},
$$

for all $s \in\left[2,2^{*}\right]$ if $n>2$ and $s \in[2,+\infty[$ if $n=2$.

Then, choosing $s=2^{*}$ if $n>2$ and $s>\max \left\{2, p_{1}\right\}$ if $n=2$, we obtain by the interpolation inequality,

$$
\begin{aligned}
& \left\|\mathcal{T}_{1}^{\varepsilon}\left(u_{1}^{\varepsilon}\right)-u_{1}\right\|_{L^{t}\left(\Omega \times Y_{1}\right)} \leq C \| \mathcal{T}_{1}^{\varepsilon}\left(u_{1}^{\varepsilon}\right) \\
& \quad-u_{1}\left\|_{L^{s}\left(\Omega \times Y_{1}\right)}^{1-a}\right\| \mathcal{T}_{1}^{\varepsilon}\left(u_{1}^{\varepsilon}\right)-u_{1} \|_{L^{2}\left(\Omega \times Y_{1}\right)}^{a} \quad \forall t \in[2, s[,
\end{aligned}
$$


with $\left.\left.a=\frac{2(s-t)}{t(s-2)} \in\right] 0,1\right]$. Hence, combining (4.1)1, (4.13) and (4.14), we get, up to a subsequence,

$$
\mathcal{T}_{1}^{\varepsilon}\left(u_{1}^{\varepsilon}\right) \longrightarrow u_{1} \quad \text { strongly in } L^{t}\left(\Omega \times Y_{1}\right) \quad \forall t \in[2, s[,
$$

for the above choice of $s$.

Now, in (4.15) we choose $t=\max \left\{2, p_{1}\right\}$ if $n=2$ and $t \in$ $\left[\max \left\{2, \frac{n+2}{n-2}\right\}, 2^{*}\left[\right.\right.$ if $n>2$. This means that $p_{1} \leq t$. Since $h_{1}$ satisfies assumptions $\left(\mathcal{H}_{1}\right)$ and $\left(\mathcal{H}_{4}\right)$, from the classical results in [32] and convergence (4.15), we obtain

$$
h_{1}\left(y, \mathcal{T}_{1}^{\varepsilon}\left(u_{1}^{\varepsilon}\right)\right) \longrightarrow h_{1}\left(y, u_{1}\right) \quad \text { strongly in } L^{t / p_{1}}\left(\Omega \times Y_{1}\right),
$$

which implies (i) by Remark 4.8.

Finally, when $\gamma<1$, convergence (ii) is a direct consequence of Theorem 4.5 and the growth condition $\left(\mathcal{H}_{4}^{\prime}\right)$ on $h_{2}$.

Remark 4.9. Due to the disconnectedness of the set $\Omega_{2}^{\varepsilon}$, we cannot obtain for $v \in V_{2}^{\varepsilon}$ a similar result as that in Lemma 4.6. This is shown by the following counter-example, suggested by Mardare [35]:

Let us fix $k_{0} \in \widehat{K}_{\varepsilon}$ and set

$$
v= \begin{cases}\varepsilon^{-n / 2} & \text { in } \varepsilon Y_{2}^{k_{0}}, \\ 0 & \text { elsewhere. }\end{cases}
$$

Then, if $s>2$,

$$
\|v\|_{L^{s}\left(\Omega_{2}^{\varepsilon}\right)}=\varepsilon^{-\frac{n}{2}}\left|\varepsilon Y_{2}^{k_{0}}\right|^{1 / s}=\varepsilon^{\frac{n}{s}-\frac{n}{2}}\left|Y_{2}^{k_{0}}\right|^{1 / s} \rightarrow+\infty, \quad \text { as } \varepsilon \rightarrow 0,
$$

while

$$
\|v\|_{H^{1}\left(\Omega_{2}^{\varepsilon}\right)}=\|v\|_{L^{2}\left(\Omega_{2}^{\varepsilon}\right)}=\left|Y_{2}^{k_{0}}\right|^{1 / s}
$$

\section{Homogenization results}

Here, we prove in details the result for $\gamma=-1$, which is more complicated. For the other cases we only clarify the points where the proof is different.

\subsection{The case $\gamma=-1$}

In this case, although the strong convergence of $\mathcal{T}_{2}^{\varepsilon}\left(u_{2}^{\varepsilon}\right)$ proved in Theorem 4.5 allows to pass to the limit in the nonlinear term $h_{2}$, we meet a main difficulty concerning the nonlinear function $h$ of the jump in the equation. Indeed, from (4.4) we have only weak convergence of the term $\left(\mathcal{T}_{1}\left(u_{1}^{\varepsilon}\right)-\mathcal{T}_{2}\left(u_{2}^{\varepsilon}\right)\right) / \varepsilon$ in $L^{2}(\Omega \times \Gamma)$ and this does not allow to pass straightforward to the limit in the interface term, as done in [26] for a linear $h$.

Nevertheless, in the spirit of the Minty method for monotone operators, by choosing a sequence of suitable test functions, we are able to compute the limit of the unfolded term $h\left(y, \mathcal{T}_{b}^{\varepsilon}\left(u_{1}^{\varepsilon}-u_{2}^{\varepsilon}\right) / \varepsilon\right)$, although we cannot improve the weak convergence of $\mathcal{T}_{b}^{\varepsilon}\left(u_{1}^{\varepsilon}-u_{2}^{\varepsilon}\right) / \varepsilon$. 
Theorem 5.1. For $\gamma=-1$, let (2.4), (2.5) and (2.6) $)_{2}$ hold true. If $u^{\varepsilon}=$ $\left(u_{1}^{\varepsilon}, u_{2}^{\varepsilon}\right)$ is the solution of problem (2.2), then there exist $u_{1} \in H_{0}^{1}(\Omega), \widehat{u}_{1} \in$ $L^{2}\left(\Omega, H_{p e r}^{1}\left(Y_{1}\right)\right)$ and $\widehat{u}_{2} \in L^{2}\left(\Omega, H^{1}\left(Y_{2}\right)\right)$ such that

$$
\begin{cases}\mathcal{T}_{1}^{\varepsilon}\left(u_{1}^{\varepsilon}\right) \longrightarrow u_{1} & \text { strongly in } L^{2}\left(\Omega, H^{1}\left(Y_{1}\right)\right), \\ \mathcal{T}_{1}^{\varepsilon}\left(\nabla u_{1}^{\varepsilon}\right) \rightarrow \nabla u_{1}+\nabla_{y} \widehat{u}_{1} & \text { weakly in } L^{2}\left(\Omega \times Y_{1}\right), \\ \mathcal{T}_{2}^{\varepsilon}\left(u_{2}^{\varepsilon}\right) \longrightarrow u_{1} & \text { strongly in } L^{2}\left(\Omega, H^{1}\left(Y_{2}\right)\right), \\ \mathcal{T}_{2}^{\varepsilon}\left(\nabla u_{2}^{\varepsilon}\right) \rightarrow \nabla u_{1}+\nabla_{y} \widehat{u}_{2} & \text { weakly in } L^{2}\left(\Omega \times Y_{2}\right) .\end{cases}
$$

The triplet $\left(u_{1}, \widehat{u}_{1}, \widehat{u}_{2}\right)$ is the unique solution of the following problem:

$$
\left\{\begin{array}{l}
\text { Find } u_{1} \in H_{0}^{1}(\Omega), \widehat{u}_{1} \in L^{2}\left(\Omega, H_{\text {per }}^{1}\left(Y_{1}\right)\right) \text { with } \mathcal{M}_{\Gamma}\left(\widehat{u}_{1}\right)=0 \\
\text { and } \widehat{u}_{2} \in L^{2}\left(\Omega, H^{1}\left(Y_{2}\right)\right) \text { such that } \\
\frac{1}{|Y|} \sum_{i=1,2} \int_{\Omega \times Y_{i}} A(y)\left(\nabla u_{1}+\nabla_{y} \widehat{u}_{i}\right)\left(\nabla \varphi+\nabla_{y} \Phi_{i}\right) d x d y+\frac{1}{|Y|} \sum_{i=1,2} \\
\quad \int_{\Omega \times Y_{i}} \varphi h_{i}\left(y, u_{1}\right) d x d y+\frac{1}{|Y|} \int_{\Omega \times \Gamma} h\left(y, \widehat{u}_{1}-\widehat{u}_{2}\right)\left(\Phi_{1}-\Phi_{2}\right) d x d \sigma_{y} \\
=\int_{\Omega} f(x) \varphi(x) d x \quad \forall \varphi \in H_{0}^{1}(\Omega), \Phi_{1} \in L^{2}\left(\Omega, H_{p e r}^{1}\left(Y_{1}\right)\right) \\
\quad \Phi_{2} \in L^{2}\left(\Omega, H^{1}\left(Y_{2}\right)\right) .
\end{array}\right.
$$

Remark 5.2. Observe that in view of Theorem 2.6, assumption (2.5) and the strict monotonicity $(2.6)_{2}$ of $h$ imply that the variational problem (2.10) admits a unique solution $u^{\varepsilon} \in H_{\gamma}^{\varepsilon}$. Besides, Proposition 2.7 gives the boundedness of the norm of $u^{\varepsilon}$ in $H_{\gamma}^{\varepsilon}$.

In the proof of Theorem 5.1, we will need the following result:

Lemma 5.3. Let the spaces $W_{\text {per }}\left(Y_{1}\right)$ and $\mathcal{B}$ defined by

$$
\begin{aligned}
W_{\text {per }}\left(Y_{1}\right) & =\left\{g \in H_{\text {per }}^{1}\left(Y_{1}\right) \mid \mathcal{M}_{\Gamma}(g)=0\right\} \\
\mathcal{B} & =H_{0}^{1}(\Omega) \times L^{2}\left(\Omega, W_{\text {per }}\left(Y_{1}\right)\right) \times L^{2}\left(\Omega, H^{1}\left(Y_{2}\right)\right) .
\end{aligned}
$$

Then, $\|\cdot\|_{\mathcal{B}}$ given by the formula

$$
\begin{aligned}
\|v\|_{\mathcal{B}}^{2}= & \left\|\nabla v_{1}+\nabla_{y} \widehat{v}_{1}\right\|_{L^{2}\left(\Omega \times Y_{1}\right)}^{2}+\left\|\nabla v_{1}+\nabla_{y} \widehat{v}_{2}\right\|_{L^{2}\left(\Omega \times Y_{2}\right)}^{2} \\
& +\left\|\widehat{v}_{1}-\widehat{v}_{2}\right\|_{L^{2}(\Omega \times \Gamma)}^{2}, v=\left(v_{1}, \widehat{v}_{1}, \widehat{v}_{2}\right) \in \mathcal{B}
\end{aligned}
$$

is a norm on $\mathcal{B}$.

Proof. Let us prove that if $\|v\|_{\mathcal{B}}=0$ then $v \equiv 0$, the remaining properties of a norm being trivial. Suppose then that $\|v\|_{\mathcal{B}}=0$. We have

$$
\left\{\begin{array}{l}
\widehat{v}_{1}=\widehat{v}_{2} \text { on } \Omega \times \Gamma, \\
M=\left\|\nabla v_{1}+\nabla_{y} \widehat{v}_{1}\right\|_{L^{2}\left(\Omega \times Y_{1}\right)}^{2}+\left\|\nabla v_{1}+\nabla_{y} \widehat{v}_{2}\right\|_{L^{2}\left(\Omega \times Y_{2}\right)}^{2}=0 .
\end{array}\right.
$$


It is easy to see that

$$
\begin{aligned}
M= & |Y|\left\|\nabla v_{1}\right\|_{L^{2}(\Omega)}^{2}+\left\|\nabla_{y} \widehat{v}_{1}\right\|_{L^{2}\left(\Omega \times Y_{1}\right)}^{2}+\left\|\nabla_{y} \widehat{v}_{2}\right\|_{L^{2}\left(\Omega \times Y_{2}\right)}^{2} \\
& +2 \int_{\Omega \times Y_{1}} \nabla v_{1} \nabla_{y} \widehat{v}_{1} d x d y+2 \int_{\Omega \times Y_{2}} \nabla v_{1} \nabla_{y} \widehat{v}_{2} d x d y .
\end{aligned}
$$

Applying Green's formula, we deduce from (5.3) 1 and the $Y$-periodicity of $\widehat{v}_{1}$

$$
\begin{aligned}
& \int_{\Omega \times Y_{1}} \nabla v_{1} \nabla_{y} \widehat{v}_{1} d x d y+\int_{\Omega \times Y_{2}} \nabla v_{1} \nabla_{y} \widehat{v}_{2} d x d y \\
& =\sum_{i=1}^{n} \int_{\Omega} \frac{\partial v_{1}}{\partial x_{i}}\left(\int_{Y_{1}} \frac{\partial \widehat{v}_{1}}{\partial y_{i}} d y\right) d x+\sum_{i=1}^{n} \int_{\Omega} \frac{\partial v_{1}}{\partial x_{i}}\left(\int_{Y_{2}} \frac{\partial \widehat{v}_{2}}{\partial y_{i}} d y\right) d x \\
& =\int_{\Omega \times \Gamma} \widehat{v}_{1} \nabla v_{1} \cdot n_{1}(y) d x d \sigma_{y}+\int_{\Omega \times \Gamma} \widehat{v}_{2} \nabla v_{1} \cdot n_{2}(y) d x d \sigma_{y} \\
& =\int_{\Omega \times \Gamma}\left(\widehat{v}_{1}-\widehat{v}_{2}\right) \nabla v_{1} \cdot n_{1}(y) d x d \sigma_{y}=0
\end{aligned}
$$

since $\nabla v_{1}$ is independent of $y$ and $n_{2}=-n_{1}$.

Combining (5.3)-(5.5) implies

$$
\begin{cases}\nabla v_{1}=0 & \text { in } \Omega \\ \nabla_{y} \widehat{v}_{1}=0 & \text { in } \Omega \times Y_{1} \\ \nabla_{y} \widehat{v}_{2}=0 & \text { in } \Omega \times Y_{2} \\ \widehat{v}_{1}=\widehat{v}_{2} & \text { on } \Omega \times \Gamma\end{cases}
$$

This means that

$$
\begin{cases}v_{1}=0 & \text { in } \Omega, \\ \widehat{v}_{1}=0 & \text { in } \Omega \times Y_{1}, \\ \widehat{v}_{2}=C(x) & \text { in every connected component of } \Omega \times Y_{2}, \\ \widehat{v}_{1}=\widehat{v}_{2} & \text { on } \Omega \times \Gamma,\end{cases}
$$

where we make use of the Poincaré inequality on $H_{0}^{1}(\Omega)$ and the PoincaréWirtinger inequality on $W_{\text {per }}\left(Y_{1}\right)$. This yields $v \equiv 0$ and the proof is complete.

Proof of Theorem 5.1. The proof consists of three steps.

Step 1: Passing to the limit.

From Theorems 4.3, 4.5 and Remark 4.4, convergences (5.1) follow for a subsequence. In order to get the limit problem, we take $\left(v_{1}, v_{2}\right)$ as test functions in $(2.10)$, where

$$
v_{i}=\varphi(x)+\varepsilon \omega_{i}(x) \psi_{i}^{\varepsilon}(x) \quad \text { with } \psi_{i}^{\varepsilon}(x)=\psi_{i}(x / \varepsilon), i=1,2,
$$

for $\varphi, \omega_{i} \in \mathcal{D}(\Omega), \psi_{1} \in H_{\text {per }}^{1}\left(Y_{1}\right)$ and $\psi_{2} \in H^{1}\left(Y_{2}\right)$ (extended by $Y$-periodicity to $\mathbb{R}^{n}$ ).

Proposition $4.2(\mathrm{v})$ gives

$$
\mathcal{T}_{i}^{\varepsilon}\left(v_{i}\right) \longrightarrow \varphi \quad \text { strongly in } L^{2}\left(\Omega \times Y_{i}\right),
$$


since $v_{i}$ strongly converges to $\varphi$ in $L^{2}(\Omega)$. Let us also note that

$$
\nabla v_{i}(x)=\nabla \varphi+\varepsilon \psi_{i}(x / \varepsilon) \nabla \omega_{i}(x)+\omega_{i}(x) \nabla_{y} \psi_{i}(x / \varepsilon) .
$$

Then, if we set $\Phi_{i}(x, y)=\omega_{i}(x) \psi_{i}(y), i=1,2$, we have

$$
\begin{aligned}
\mathcal{T}_{i}^{\varepsilon}\left(\nabla v_{i}\right) & =\mathcal{T}_{i}^{\varepsilon}(\nabla \varphi)+\varepsilon \psi_{i} \mathcal{T}_{i}^{\varepsilon}\left(\nabla \omega_{i}\right)+\nabla_{y} \psi_{i} \mathcal{T}_{i}^{\varepsilon}\left(\omega_{i}\right) \\
& \longrightarrow \nabla \varphi+\nabla_{y} \Phi_{i} \quad \text { strongly in } L^{2}\left(\Omega \times Y_{i}\right) .
\end{aligned}
$$

Hence, from convergences $(5.1)_{2,4},(5.8),(5.9)$ and Proposition 4.7, we obtain by unfolding,

$$
\begin{aligned}
\int_{\Omega_{i}^{\varepsilon}} A^{\varepsilon} \nabla u_{i}^{\varepsilon} \nabla v_{i} d x & =\frac{1}{|Y|} \int_{\Omega \times Y_{i}} A(y) \mathcal{T}_{i}^{\varepsilon}\left(\nabla u_{i}^{\varepsilon}\right) \mathcal{T}_{i}^{\varepsilon}\left(\nabla v_{i}\right) d x d y \\
& \longrightarrow \frac{1}{|Y|} \int_{\Omega \times Y_{i}} A(y)\left(\nabla u_{1}+\nabla_{y} \widehat{u}_{i}\right)\left(\nabla \varphi+\nabla_{y} \Phi_{i}\right) d x d y \\
\int_{\Omega_{i}^{\varepsilon}} h_{i}^{\varepsilon}\left(x, u_{i}^{\varepsilon}\right) v_{i} d x & =\frac{1}{|Y|} \int_{\Omega \times Y_{i}} h_{i}\left(y, \mathcal{T}_{i}^{\varepsilon}\left(u_{i}^{\varepsilon}\right)\right) \mathcal{T}_{i}^{\varepsilon}\left(v_{i}\right) d x d y \\
& \longrightarrow \frac{1}{|Y|} \int_{\Omega \times Y_{i}} h_{i}\left(y, u_{1}\right) \varphi d x d y
\end{aligned}
$$

for $i=1,2$ and

$$
\int_{\Omega_{1}^{\varepsilon}} f v_{1} d x+\int_{\Omega_{2}^{\varepsilon}} f v_{2} d x \longrightarrow \int_{\Omega} \theta_{1} f \varphi d x+\int_{\Omega} \theta_{2} f \varphi d x=\int_{\Omega} f \varphi d x
$$

On the other hand, in view of Remark 4.8,

$$
\begin{aligned}
& \int_{\Gamma^{\varepsilon}} h^{\varepsilon}\left(x, u_{1}^{\varepsilon}-u_{2}^{\varepsilon}\right)\left(v_{1}-v_{2}\right) d \sigma_{x}=\varepsilon \int_{\Gamma^{\varepsilon}} h^{\varepsilon}\left(x, u_{1}^{\varepsilon}-u_{2}^{\varepsilon}\right)\left(\omega_{1} \psi_{1}^{\varepsilon}-\omega_{2} \psi_{2}^{\varepsilon}\right) d \sigma_{x} \\
& =\frac{1}{|Y|} \int_{\Omega \times \Gamma} h\left(y, \frac{\mathcal{T}_{b}^{\varepsilon}\left(u_{1}^{\varepsilon}-u_{2}^{\varepsilon}\right)}{\varepsilon}\right)\left(\psi_{1}(y) \mathcal{T}_{1}^{\varepsilon}\left(\omega_{1}\right)-\psi_{2}(y) \mathcal{T}_{2}^{\varepsilon}\left(\omega_{2}\right)\right) d x d \sigma_{y}
\end{aligned}
$$

Note that Theorem 4.3 implies

$$
\left\|\frac{\mathcal{T}_{b}^{\varepsilon}\left(u_{1}^{\varepsilon}-u_{2}^{\varepsilon}\right)}{\varepsilon}\right\|_{L^{2}(\Omega \times \Gamma)} \leq c .
$$

Thus, in view of assumption $\left(\mathcal{H}_{2}\right)$ on $h$ and the fact that $q<2$, there exists $\zeta \in L^{2 / q}(\Omega \times \Gamma)$ such that, up to a subsequence of $\varepsilon$,

$$
h\left(y, \frac{\mathcal{T}_{b}^{\varepsilon}\left(u_{1}^{\varepsilon}-u_{2}^{\varepsilon}\right)}{\varepsilon}\right) \rightarrow \zeta \quad \text { weakly in } L^{2 / q}(\Omega \times \Gamma) .
$$

Then, we obtain

$$
\lim _{\varepsilon \rightarrow 0} \int_{\Gamma^{\varepsilon}} h^{\varepsilon}\left(x, u_{1}^{\varepsilon}-u_{2}^{\varepsilon}\right)\left(v_{1}-v_{2}\right) d \sigma_{x}=\frac{1}{|Y|} \int_{\Omega \times \Gamma} \zeta\left(\Phi_{1}-\Phi_{2}\right) d x d \sigma_{y} .
$$


Collecting (5.10)-(5.12) and (5.15), passing to the limit as $\varepsilon \rightarrow 0$ in the variational formulation (2.10) for $\gamma=-1$ and $\left(v_{1}, v_{2}\right)$ chosen as above, by density we deduce the following limit problem:

$$
\left\{\begin{array}{l}
\frac{1}{|Y|} \sum_{i=1,2} \int_{\Omega \times Y_{i}} A(y)\left(\nabla u_{1}+\nabla_{y} \widehat{u}_{i}\right)\left(\nabla \varphi+\nabla_{y} \Phi_{i}\right) d x d y \\
+\frac{1}{|Y|} \int_{\Omega \times \Gamma} \zeta\left(\Phi_{1}-\Phi_{2}\right) d x d \sigma_{y}+\frac{1}{|Y|} \sum_{i=1,2} \int_{\Omega \times Y_{i}} \varphi h_{i}\left(y, u_{1}\right) d x d y \\
=\int_{\Omega} f(x) \varphi(x) d x \quad \forall \varphi \in H_{0}^{1}(\Omega), \Phi_{1} \in L^{2}\left(\Omega, H_{p e r}^{1}\left(Y_{1}\right)\right) \\
\Phi_{2} \in L^{2}\left(\Omega, H^{1}\left(Y_{2}\right)\right) .
\end{array}\right.
$$

Step 2: Identification of the limit $\zeta$ in (5.16).

We now show that

$$
\zeta=h\left(y, \widehat{u}_{1}-\widehat{u}_{2}\right) .
$$

In order to do that, we use the Minty method for monotone operators, as done for instance in [19] for Leray-Lions operators by the unfolding method or in [3] for a parabolic problem with a jump of solution by the two-scale method.

This leads to use some test functions adapted to our nonlinear problem. Consider the sequences

$$
\varpi_{i}^{\varepsilon}(x)=\lambda_{0}(x)+\varepsilon \lambda_{i 1}\left(x, \frac{x}{\varepsilon}\right)+k \varepsilon \lambda_{i 2}\left(x, \frac{x}{\varepsilon}\right), \quad \text { for } i=1,2,
$$

where

$$
\left\{\begin{array}{l}
\lambda_{0} \in \mathcal{D}(\Omega), \lambda_{1 j} \in D\left(\Omega, C_{p e r}^{\infty}\left(Y_{1}\right)\right) \\
\lambda_{2 j} \in D\left(\Omega, C^{\infty}\left(Y_{2}\right)\right) \text { extended by Y-periodicity to } \mathbb{R}^{n}, j=1,2 .
\end{array}\right.
$$

First, taking $\left(u_{1}^{\varepsilon}-\varpi_{1}^{\varepsilon}, u_{2}^{\varepsilon}-\varpi_{2}^{\varepsilon}\right)$ as a test function in (2.10) written for $\gamma=-1$ implies

$$
\begin{aligned}
& \sum_{i=1,2} \int_{\Omega_{i}^{\varepsilon}} A^{\varepsilon} \nabla u_{i}^{\varepsilon}\left(\nabla u_{i}^{\varepsilon}-\nabla \varpi_{i}^{\varepsilon}\right) d x+\sum_{i=1,2} \int_{\Omega_{i}^{\varepsilon}}\left(u_{i}^{\varepsilon}-\varpi_{i}^{\varepsilon}\right) h_{i}^{\varepsilon}\left(x, u_{i}^{\varepsilon}\right) d x \\
& \quad+\int_{\Gamma^{\varepsilon}}\left(u_{1}^{\varepsilon}-\varpi_{1}^{\varepsilon}-u_{2}^{\varepsilon}+\varpi_{2}^{\varepsilon}\right) h^{\varepsilon}\left(x, u_{1}^{\varepsilon}-u_{2}^{\varepsilon}\right) d \sigma_{x}=\sum_{i=1,2} \int_{\Omega_{i}^{\varepsilon}} f\left(u_{i}^{\varepsilon}-\varpi_{i}^{\varepsilon}\right) d x .
\end{aligned}
$$

Moreover, from the monotonicity of $h, h_{1}, h_{2}$ and the assumption $A \in$ $M(\alpha, \beta, Y)$ we have

$$
\begin{aligned}
& \sum_{i=1,2} \int_{\Omega_{i}^{\varepsilon}} A^{\varepsilon}\left(\nabla u_{i}^{\varepsilon}-\nabla \varpi_{i}^{\varepsilon}\right)\left(\nabla u_{i}^{\varepsilon}-\nabla \varpi_{i}^{\varepsilon}\right) d x \\
& \quad+\sum_{i=1,2} \int_{\Omega_{i}^{\varepsilon}}\left(u_{i}^{\varepsilon}-\varpi_{i}^{\varepsilon}\right)\left[h_{i}^{\varepsilon}\left(x, u_{i}^{\varepsilon}\right)-h_{i}^{\varepsilon}\left(x, \varpi_{i}^{\varepsilon}\right)\right] d x \\
& \quad+\int_{\Gamma^{\varepsilon}}\left(u_{1}^{\varepsilon}-u_{2}^{\varepsilon}-\varpi_{1}^{\varepsilon}+\varpi_{2}^{\varepsilon}\right)\left[h^{\varepsilon}\left(x, u_{1}^{\varepsilon}-u_{2}^{\varepsilon}\right)-h^{\varepsilon}\left(x, \varpi_{1}^{\varepsilon}-\varpi_{2}^{\varepsilon}\right)\right] d \sigma_{x} \geq 0 .
\end{aligned}
$$


Thus, combining (5.19) with (5.20) yields

$$
\begin{aligned}
& \sum_{i=1,2} \int_{\Omega_{i}^{\varepsilon}} A^{\varepsilon} \nabla \varpi_{i}^{\varepsilon}\left(\nabla u_{i}^{\varepsilon}-\nabla \varpi_{i}^{\varepsilon}\right) d x+\sum_{i=1,2} \int_{\Omega_{i}^{\varepsilon}}\left(u_{i}^{\varepsilon}-\varpi_{i}^{\varepsilon}\right) h_{i}^{\varepsilon}\left(x, \varpi_{i}^{\varepsilon}\right) d x \\
& \quad+\int_{\Gamma^{\varepsilon}}\left(u_{1}^{\varepsilon}-u_{2}^{\varepsilon}-\varpi_{1}^{\varepsilon}+\varpi_{2}^{\varepsilon}\right) h^{\varepsilon}\left(x, \varpi_{1}^{\varepsilon}-\varpi_{2}^{\varepsilon}\right) d \sigma_{x} \leq \sum_{i=1,2} \int_{\Omega_{i}^{\varepsilon}} f\left(u_{i}^{\varepsilon}-\varpi_{i}^{\varepsilon}\right) d x,
\end{aligned}
$$

which gives, by unfolding, for $\varepsilon$ small enough

$$
\begin{aligned}
& \frac{1}{|Y|} \sum_{i=1,2} \int_{\Omega \times Y_{i}} A(y) \mathcal{T}_{i}^{\varepsilon}\left(\nabla \varpi_{i}^{\varepsilon}\right)\left[\mathcal{T}_{i}^{\varepsilon}\left(\nabla u_{i}^{\varepsilon}\right)-\mathcal{T}_{i}^{\varepsilon}\left(\nabla \varpi_{i}^{\varepsilon}\right)\right] d x d y \\
& \quad+\frac{1}{|Y|} \sum_{i=1,2} \int_{\Omega \times Y_{i}} \mathcal{T}_{i}^{\varepsilon}\left(u_{i}^{\varepsilon}-\varpi_{i}^{\varepsilon}\right) h_{i}\left(y, \mathcal{T}_{i}^{\varepsilon}\left(\varpi_{i}^{\varepsilon}\right)\right) d x d y \\
& \quad+\frac{1}{|Y|} \int_{\Omega \times \Gamma}\left[\frac{\mathcal{T}_{b}^{\varepsilon}\left(u_{1}^{\varepsilon}-u_{2}^{\varepsilon}\right)}{\varepsilon}-\frac{\mathcal{T}_{b}^{\varepsilon}\left(\varpi_{1}^{\varepsilon}-\varpi_{2}^{\varepsilon}\right)}{\varepsilon}\right] h\left(y, \frac{\mathcal{T}_{b}^{\varepsilon}\left(\varpi_{1}^{\varepsilon}-\varpi_{2}^{\varepsilon}\right)}{\varepsilon}\right) d x d \sigma_{y} \\
& \leq \frac{1}{|Y|} \sum_{i=1,2} \int_{\Omega \times Y_{i}} \mathcal{T}_{i}^{\varepsilon}(f) \mathcal{T}_{i}^{\varepsilon}\left(u_{i}^{\varepsilon}-\varpi_{i}^{\varepsilon}\right) d x d y .
\end{aligned}
$$

On the other hand, it is easily seen that, as $\varepsilon \rightarrow 0$

$$
\left\{\begin{array}{l}
\mathcal{T}_{i}^{\varepsilon}\left(\varpi_{i}^{\varepsilon}\right) \longrightarrow \lambda_{0} \text { strongly in } L^{2}\left(\Omega \times Y_{i}\right), \\
\frac{1}{\varepsilon} \mathcal{T}_{i}^{\varepsilon}\left(\varepsilon \lambda_{i 1}\left(x, \frac{x}{\varepsilon}\right)+k \varepsilon \lambda_{i 2}\left(x, \frac{x}{\varepsilon}\right)\right) \longrightarrow \lambda_{i 1}(x, y)+k \lambda_{i 2}(x, y) \\
\text { strongly in } L^{2}\left(\Omega, H^{1}\left(Y_{i}\right)\right) \text { and } L^{2}(\Omega \times \Gamma), \text { for } i=1,2 .
\end{array}\right.
$$

Consequently,

$$
\frac{\mathcal{T}_{b}^{\varepsilon}\left(\varpi_{1}^{\varepsilon}-\varpi_{2}^{\varepsilon}\right)}{\varepsilon} \longrightarrow \lambda_{11}-\lambda_{21}+k\left(\lambda_{12}-\lambda_{22}\right) \quad \text { strongly in } L^{2}(\Omega \times \Gamma) .
$$

Moreover, for $i=1,2$, since

$$
\begin{aligned}
\nabla \varpi_{i}^{\varepsilon}(x)= & \nabla \lambda_{0}(x)+\varepsilon \nabla \lambda_{i 1}\left(x, \frac{x}{\varepsilon}\right)+\nabla_{y} \lambda_{i 1}\left(x, \frac{x}{\varepsilon}\right) \\
& +k \varepsilon \nabla \lambda_{i 2}\left(x, \frac{x}{\varepsilon}\right)+k \nabla_{y} \lambda_{i 2}\left(x, \frac{x}{\varepsilon}\right),
\end{aligned}
$$

we obtain

$\mathcal{T}_{i}^{\varepsilon}\left(\nabla \varpi_{i}^{\varepsilon}\right) \longrightarrow \nabla \lambda_{0}(x)+\nabla_{y} \lambda_{i 1}(x, y)+k \nabla_{y} \lambda_{i 2}(x, y) \quad$ strongly in $L^{2}\left(\Omega \times Y_{i}\right)$

Thus, passing to the limit in (5.22) as $\varepsilon \rightarrow 0$, one deduces

$$
\begin{aligned}
& \sum_{i=1,2} \int_{\Omega \times Y_{i}} A(y)\left(\nabla \lambda_{0}+\nabla_{y} \lambda_{i 1}+k \nabla_{y} \lambda_{i 2}\right)\left[\nabla u_{1}+\nabla_{y} \widehat{u}_{i}\right. \\
& \left.\quad-\left(\nabla \lambda_{0}+\nabla_{y} \lambda_{i 1}+k \nabla_{y} \lambda_{i 2}\right)\right] d x d y
\end{aligned}
$$




$$
\begin{aligned}
& +\int_{\Omega \times \Gamma}\left[\widehat{u}_{1}-\widehat{u}_{2}-\lambda_{11}+\lambda_{21}-k\left(\lambda_{12}-\lambda_{22}\right)\right] h\left(y, \lambda_{11}-\lambda_{21}+k\left(\lambda_{12}-\lambda_{22}\right)\right) d x d \sigma_{y} \\
& +\sum_{i=1,2} \int_{\Omega \times Y_{i}}\left(u_{1}-\lambda_{0}\right) h_{i}\left(y, \lambda_{0}\right) d x d y \leq|Y| \int_{\Omega} f\left(u_{1}-\lambda_{0}\right) d x d y,
\end{aligned}
$$

where we use (5.23), (5.24) and the convergence results in Theorem 4.3.

Now let us choose $\lambda_{0}=\lambda_{0}^{n}$ and $\lambda_{i 1}=\lambda_{i 1}^{n}, i=1,2$ such that, as $n \rightarrow+\infty$

$$
\begin{cases}\lambda_{0}^{n} \longrightarrow u_{1} & \text { strongly in } H_{0}^{1}(\Omega) \\ \lambda_{11}^{n} \longrightarrow \widehat{u}_{1} & \text { strongly in } L^{2}\left(\Omega, H_{p e r}^{1}\left(Y_{1}\right)\right) \\ \lambda_{21}^{n} \longrightarrow \widehat{u}_{2} & \text { strongly in } L^{2}\left(\Omega, H^{1}\left(Y_{2}\right)\right)\end{cases}
$$

and pass to the limit as $n \rightarrow+\infty$ in (5.25), we obtain

$$
\begin{aligned}
& -k \sum_{i=1,2} \int_{\Omega \times Y_{i}} A(y)\left(\nabla u_{1}+\nabla_{y} \widehat{u}_{i}+k \nabla_{y} \lambda_{i 2}\right) \nabla_{y} \lambda_{i 2} d x d y \\
& -k \int_{\Omega \times \Gamma}\left(\lambda_{12}-\lambda_{22}\right) h\left(y, \widehat{u}_{1}-\widehat{u}_{2}+k\left(\lambda_{12}-\lambda_{22}\right)\right) d x d \sigma_{y} \leq 0 .
\end{aligned}
$$

Since (5.27) holds for every $k \in \mathbb{R}$, we deduce the following equality:

$$
\begin{aligned}
& \sum_{i=1,2} \int_{\Omega \times Y_{i}} A(y)\left(\nabla u_{1}+\nabla_{y} \widehat{u}_{i}+k \nabla_{y} \lambda_{i 2}\right) \nabla_{y} \lambda_{i 2} d x d y \\
& \quad+\int_{\Omega \times \Gamma}\left(\lambda_{12}-\lambda_{22}\right) h\left(y, \widehat{u}_{1}-\widehat{u}_{2}+k\left(\lambda_{12}-\lambda_{22}\right)\right) d x d \sigma_{y}=0 .
\end{aligned}
$$

On the other hand, choosing $\varphi \equiv 0, \Phi_{1}=\lambda_{12}, \Phi_{2}=\lambda_{22}$ as the test functions in (5.16) implies

$$
\sum_{i=1,2} \int_{\Omega \times Y_{i}} A(y)\left(\nabla u_{1}+\nabla_{y} \widehat{u}_{i}\right) \nabla_{y} \lambda_{i 2} d x d y+\int_{\Omega \times \Gamma} \zeta\left(\lambda_{12}-\lambda_{22}\right) d x d \sigma_{y}=0 .
$$

Combining (5.28) with (5.29), one gets

$$
\begin{aligned}
k & \sum_{i=1,2} \int_{\Omega \times Y_{i}} A(y) \nabla_{y} \lambda_{i 2} \nabla_{y} \lambda_{i 2} d x d y-\int_{\Omega \times \Gamma} \zeta\left(\lambda_{12}-\lambda_{22}\right) d x d \sigma_{y} \\
& +\int_{\Omega \times \Gamma}\left(\lambda_{12}-\lambda_{22}\right) h\left(y, \widehat{u}_{1}-\widehat{u}_{2}+k\left(\lambda_{12}-\lambda_{22}\right)\right) d x d \sigma_{y}=0 .
\end{aligned}
$$

Thus, letting $k \rightarrow 0$ in $(5.30)$ provides

$$
\int_{\Omega \times \Gamma}\left(\lambda_{12}-\lambda_{22}\right) h\left(y, \widehat{u}_{1}-\widehat{u}_{2}\right) d x d \sigma_{y}=\int_{\Omega \times \Gamma} \zeta\left(\lambda_{12}-\lambda_{22}\right) d x d \sigma_{y}
$$

which gives (5.17).

Step 3: Existence and uniqueness of the solution of the limit problem (5.2). 
Let us consider operator $\mathcal{P}: u=\left(u_{1}, \widehat{u}_{1}, \widehat{u}_{2}\right) \in \mathcal{B} \longmapsto \mathcal{P}(u) \in \mathcal{B}^{\prime}$ defined by

$$
\begin{aligned}
& \langle\mathcal{P}(u), v\rangle_{\mathcal{B}^{\prime}, \mathcal{B}}=\sum_{i=1,2} \int_{\Omega \times Y_{i}} A(y)\left(\nabla u_{1}+\nabla_{y} \widehat{u}_{i}\right)\left(\nabla v_{1}+\nabla_{y} \widehat{v}_{i}\right) d x d y \\
& \quad+\sum_{i=1,2} \int_{\Omega \times Y_{i}} v_{1} h_{i}\left(y, u_{1}\right) d x d y \\
& \quad+\int_{\Omega \times \Gamma} h\left(y, \widehat{u}_{1}-\widehat{u}_{2}\right)\left(\widehat{v}_{1}-\widehat{v}_{2}\right) d x d \sigma_{y}-|Y| \int_{\Omega} f v_{1} d x \quad \forall v=\left(v_{1}, \widehat{v}_{1}, \widehat{v}_{2}\right) \in \mathcal{B},
\end{aligned}
$$

where the space $\mathcal{B}$ is given in Lemma 5.3.

It is easily seen that $\mathcal{P}$ is bounded continuous, coercive and monotone. Thus, from the Minty-Browder theorem, $\mathcal{P}$ is surjective, which implies that Eq. (5.2) has a solution $u=\left(u_{1}, \widehat{u}_{1}, \widehat{u}_{2}\right) \in \mathcal{B}$. Let us check the uniqueness of $u$. If problem (5.2) has two solutions $u=\left(u_{1}, \widehat{u}_{1}, \widehat{u}_{2}\right)$ and $\omega=\left(\omega_{1}, \widehat{\omega}_{1}, \widehat{\omega}_{2}\right)$, we have

$$
\langle\mathcal{P}(u)-\mathcal{P}(\omega), v\rangle_{\mathcal{B}^{\prime}, \mathcal{B}}=0 \quad \forall v=\left(v_{1}, \widehat{v}_{1}, \widehat{v}_{2}\right) \in \mathcal{B}
$$

Choosing $v=u-\omega$ yields

$$
\begin{aligned}
& \langle\mathcal{P}(u)-\mathcal{P}(\omega), u-\omega\rangle_{\mathcal{B}^{\prime}, \mathcal{B}} \\
& =\sum_{i=1,2} \int_{\Omega \times Y_{i}} A(y)\left(\nabla u_{1}-\nabla \omega_{1}+\nabla_{y} \widehat{u}_{i}-\nabla_{y} \widehat{\omega}_{i}\right) \\
& \quad \times\left(\nabla u_{1}-\nabla \omega_{1}+\nabla_{y} \widehat{u}_{i}-\nabla_{y} \widehat{\omega}_{i}\right) d x d y \\
& \quad+\sum_{i=1,2} \int_{\Omega \times Y_{i}}\left(h_{i}\left(y, u_{1}\right)-h_{i}\left(y, \omega_{1}\right)\right)\left(u_{1}-\omega_{1}\right) d x d y \\
& \quad+\int_{\Omega \times \Gamma}\left(h\left(y, \widehat{u}_{1}-\widehat{u}_{2}\right)-h\left(y, \widehat{\omega}_{1}-\widehat{\omega}_{2}\right)\right)\left(\widehat{u}_{1}-\widehat{u}_{2}-\widehat{\omega}_{1}+\widehat{\omega}_{2}\right) d x d \sigma_{y}=0 .
\end{aligned}
$$

Using the assumption $A \in M(\alpha, \beta, Y)$ and the strictly monotonicity of $h$, we obtain

$$
\begin{cases}\nabla\left(u_{1}-\omega_{1}\right)+\nabla_{y}\left(\widehat{u}_{i}-\widehat{\omega}_{i}\right)=0 & \text { in } \Omega \times Y_{i}, i=1,2, \\ \widehat{u}_{1}-\widehat{\omega}_{1}-\left(\widehat{u}_{2}-\widehat{\omega}_{2}\right)=0 & \text { on } \Omega \times \Gamma,\end{cases}
$$

which by Lemma 5.3 gives $\|u-\omega\|_{\mathcal{B}}=0$, so that $u \equiv \omega$. Hence, convergences (5.1) hold for the whole sequence. This completes the proof.

Remark 5.4. The fact that we cannot directly pass to the limit in the unfolded nonlinear term $h\left(y, \mathcal{T}_{b}^{\varepsilon}\left(u_{1}^{\varepsilon}-u_{2}^{\varepsilon}\right) / \varepsilon\right)$ makes the situation here different from the case of nonlinear Robin boundary conditions treated in $[7,16]$, where some weak convergence of the Nemytskii operator associated to the nonlinear function $h$ was proved. 
In order to describe the homogenized problem solved by $u_{1}$, we introduce for every $z \in \mathbb{R}^{n}$, the following problem:

$$
\left\{\begin{array}{l}
\text { Find }\left(\chi_{1}(\cdot, z), \chi_{2}(\cdot, z)\right) \in W_{p e r}\left(Y_{1}\right) \times H^{1}\left(Y_{2}\right) \text { such that } \\
\sum_{i=1,2} \int_{Y_{i}} A(y) \nabla_{y} \chi_{i}(y, z) \nabla v_{i} d y+\int_{\Gamma} h\left(y, \chi_{1}(y, z)-\chi_{2}(y, z)\right)\left(v_{1}-v_{2}\right) d \sigma \\
=-\int_{Y_{1}} A(y) z \nabla v_{1} d y-\int_{Y_{2}} A(y) z \nabla v_{2} d y, \forall v_{1} \in H_{p e r}^{1}\left(Y_{1}\right), v_{2} \in H^{1}\left(Y_{2}\right),
\end{array}\right.
$$

where the space $W_{\text {per }}\left(Y_{1}\right)$ is defined in Lemma 5.3.

The existence and the uniqueness of the solution of the above problem can be proved using the Minty-Browder theorem as in the proof of Proposition 3.2 , for the functional space

$$
W \doteq\left\{v=\left(v_{1}, v_{2}\right): v_{1} \in W_{p e r}\left(Y_{1}\right), v_{2} \in H^{1}\left(Y_{2}\right)\right\},
$$

endowed with the norm

$$
\|v\|_{W}^{2}=\left\|\nabla v_{1}\right\|_{L^{2}\left(Y_{1}\right)}^{2}+\left\|\nabla v_{2}\right\|_{L^{2}\left(Y_{2}\right)}^{2}+\left\|v_{1}-v_{2}\right\|_{L^{2}(\Gamma)}^{2} \quad \forall v=\left(v_{1}, v_{2}\right) \in W .
$$

Corollary 5.5. For $\gamma=-1$, under the hypotheses of Theorem 5.1, if $u^{\varepsilon}=$ $\left(u_{1}^{\varepsilon}, u_{2}^{\varepsilon}\right)$ is the solution of problem $(2.2)$, then there exists $u_{1} \in H_{0}^{1}(\Omega)$ such that, for $i=1,2$,

$$
\begin{cases}\widetilde{u}_{i}^{\varepsilon} \rightarrow \theta_{i} u_{1} & \text { weakly in } L^{2}(\Omega), \\ A^{\varepsilon} \widetilde{\nabla u_{i}^{\varepsilon}} \rightarrow \theta_{i} \mathcal{M}_{Y_{i}}\left(A(y)\left[\nabla u_{1}+\nabla_{y} \chi_{i}\left(y, \nabla u_{1}\right)\right]\right) & \text { weakly in }\left(L^{2}(\Omega)\right)^{n}\end{cases}
$$

and $u_{1}$ is the unique solution of the following nonlinear problem:

$$
\left\{\begin{array}{l}
-\operatorname{div}\left(D\left(\nabla u_{1}\right)\right)+\theta_{1} \mathcal{M}_{Y_{1}}\left(h_{1}\left(\cdot, u_{1}\right)\right)+\theta_{2} \mathcal{M}_{Y_{2}}\left(h_{2}\left(\cdot, u_{1}\right)\right)=f \quad \text { in } \Omega, \\
u_{1}=0 \text { on } \partial \Omega
\end{array}\right.
$$

where

$$
\begin{aligned}
D(z)= & \sum_{i=1}^{2} \theta_{i} \mathcal{M}_{Y_{i}}\left[A(y)\left(z+\nabla_{y} \chi_{i}(y, z)\right)\right]=\mathcal{M}_{Y}(A) z \\
& +\sum_{i=1}^{2} \theta_{i} \mathcal{M}_{Y_{i}}\left(A(y) \nabla_{y} \chi_{i}(y, z)\right)
\end{aligned}
$$

and for every $z \in \mathbb{R}^{n}$, the pair $\left(\chi_{1}(\cdot, z), \chi_{2}(\cdot, z)\right)$ is the unique solution of problem (5.32).

Proof. Choosing $\varphi \equiv 0$ in (5.2) yields

$$
\begin{aligned}
& \frac{1}{|Y|} \sum_{i=1,2} \int_{\Omega \times Y_{i}} A(y)\left(\nabla u_{1}+\nabla_{y} \widehat{u}_{i}\right) \nabla_{y} \Phi_{i} d x d y \\
& \quad+\frac{1}{|Y|} \int_{\Omega \times \Gamma} h\left(y, \widehat{u}_{1}-\widehat{u}_{2}\right)\left(\Phi_{1}-\Phi_{2}\right) d x d \sigma_{y}=0 .
\end{aligned}
$$


This implies that

$$
\begin{cases}-\operatorname{div}_{y}\left(A(y) \nabla_{y} \widehat{u}_{1}(x, y)\right)=\operatorname{div}_{y}\left(A(y) \nabla u_{1}(x)\right) & \text { a.e. in } \Omega \times Y_{1}, \\ -\operatorname{div}_{y}\left(A(y) \nabla_{y} \widehat{u}_{2}(x, y)\right)=\operatorname{div}_{y}\left(A(y) \nabla u_{1}(x)\right) & \text { a.e. in } \Omega \times Y_{2}, \\ A(y)\left[\nabla_{y} \widehat{u}_{1}(x, y)+\nabla u_{1}(x)\right] n_{1} & \\ \quad=-A(y)\left[\nabla_{y} \widehat{u}_{2}(x, y)+\nabla u_{1}(x)\right] n_{2} & \text { a.e. in } \Omega \times \Gamma, \\ -A(y)\left[\nabla_{y} \widehat{u}_{1}(x, y)+\nabla u_{1}(x)\right] n_{1}=h\left(y, \widehat{u}_{1}-\widehat{u}_{2}\right) & \text { a.e. in } \Omega \times \Gamma, \\ \widehat{u}_{1}(x, \cdot) Y \text {-periodic. } & \end{cases}
$$

Clearly, (5.37) provides $\left(\widehat{u}_{1}, \widehat{u}_{2}\right)$ under the following forms:

$$
\left\{\begin{array}{l}
\widehat{u}_{1}(x, y)=\chi_{1}\left(y, \nabla u_{1}\right), \\
\widehat{u}_{2}(x, y)=\chi_{2}\left(y, \nabla u_{1}\right),
\end{array}\right.
$$

where, for every $z \in \mathbb{R}^{n}$, the pair $\left(\chi_{1}(\cdot, z), \chi_{2}(\cdot, z)\right) \in W_{p e r}\left(Y_{1}\right) \times H^{1}\left(Y_{2}\right)$ is the solution of problem (5.32).

On the other hand, choosing $\Phi_{1}=\Phi_{2} \equiv 0$ in (5.2) gives

$$
\begin{aligned}
& \frac{1}{|Y|} \sum_{i=1,2} \int_{\Omega \times Y_{i}} A(y)\left(\nabla u_{1}+\nabla_{y} \widehat{u}_{i}\right) \nabla \varphi d x d y+\frac{1}{|Y|} \sum_{i=1,2} \int_{\Omega \times Y_{i}} h_{i}\left(y, u_{1}\right) \varphi d x d y \\
& \quad=\int_{\Omega} f(x) \varphi(x) d x
\end{aligned}
$$

that is

$$
\left\{\begin{array}{l}
\frac{1}{|Y|} \sum_{i=1,2} \operatorname{div}_{x} \int_{Y_{i}} A(y)\left[\nabla u_{1}(x)+\nabla_{y} \chi_{i}\left(y, \nabla u_{1}\right)\right] d y \\
\quad+\frac{1}{|Y|} \sum_{i=1,2} \int_{Y_{i}} h_{i}\left(y, u_{1}(x)\right) d y=f \quad \text { in } \Omega \\
u_{1}=0 \text { on } \partial \Omega,
\end{array}\right.
$$

where we replaced (5.38) in (5.39). This, using (5.35) gives the homogenized problem (5.34).

Let us now check the uniqueness of solution $u_{1}$ of problem (5.34). Suppose that $u_{1}^{*}$ is a solution of $(5.34)$ and set $\widehat{u}_{1}^{*}=\chi_{1}\left(y, \nabla u_{1}^{*}\right)$ and $\widehat{u}_{2}^{*}=\chi_{2}\left(y, \nabla u_{1}^{*}\right)$. Since $\left(\chi_{1}(\cdot, z), \chi_{2}(\cdot, z)\right)$ is the unique solution of problem (5.32) for every $z \in$ $\mathbb{R}^{n},\left(u_{1}^{*}, \widehat{u}_{1}^{*}, \widehat{u}_{2}^{*}\right)$ satisfies (5.36) and (5.39). Summing these two equalities, one has that $\left(u_{1}^{*}, \widehat{u}_{1}^{*}, \widehat{u}_{2}^{*}\right)$ is a solution of problem (5.2) which by Theorem 5.1 has a unique solution. Hence, $u_{1}^{*} \equiv u_{1}$ and problem (5.34) admits a unique solution.

Finally, convergences (5.33) follow from Proposition 4.2(i), (vi), (vii) together with (5.1) and (5.38).

\subsection{The case $\gamma<-1$}

In this case, as done in [26], thanks to the relationship between the traces of $\widehat{u}_{1}$ and $\widehat{u}_{2}$ on $\Gamma$ provided by Theorem 4.3 , we can overcome the difficulty given by the presence of the nonlinear jump on the boundary. 
Theorem 5.6. For $\gamma<-1$, under assumptions (2.4), (2.5) and $(2.6)_{1}$ let $u^{\varepsilon}=$ $\left(u_{1}^{\varepsilon}, u_{2}^{\varepsilon}\right)$ be the solution of problem $(2.2)$. Then, there exist $u_{1} \in H_{0}^{1}(\Omega), \widehat{u}_{1} \in$ $L^{2}\left(\Omega, H_{p e r}^{1}\left(Y_{1}\right)\right)$ and $\widehat{u}_{2} \in L^{2}\left(\Omega, H^{1}\left(Y_{2}\right)\right)$ such that convergences (5.1) hold. If $\widehat{u}$ (extended by periodicity) is given by

$$
\widehat{u}= \begin{cases}\widehat{u}_{1} & \text { in } \Omega \times Y_{1}, \\ \widehat{u}_{2}+\xi_{\Gamma} & \text { in } \Omega \times Y_{2},\end{cases}
$$

with $\xi_{\Gamma}$ as in Theorem 4.3, then the pair $\left(u_{1}, \widehat{u}\right)$ is the unique solution of the following problem:

$$
\left\{\begin{array}{l}
\text { Find } u_{1} \in H_{0}^{1}(\Omega), \widehat{u} \in L^{2}\left(\Omega, H_{\text {per }}^{1}(Y)\right) \text { with } \mathcal{M}_{\Gamma}(\widehat{u})=0 \text { a.e. in } \Omega \\
\quad \text { such that } \\
\frac{1}{|Y|} \int_{\Omega \times Y} A(y)\left(\nabla u_{1}+\nabla_{y} \widehat{u}\right)\left(\nabla \varphi+\nabla_{y} \Phi\right) d x d y \\
\quad+\frac{1}{|Y|} \sum_{i=1,2} \int_{\Omega \times Y_{i}} h_{i}\left(y, u_{1}\right) \varphi d x d y \\
=\int_{\Omega} f(x) \varphi(x) d x \quad \forall \varphi \in H_{0}^{1}(\Omega), \forall \Phi \in L^{2}\left(\Omega, H_{p e r}^{1}(Y)\right) .
\end{array}\right.
$$

Proof. Let us choose

$$
v_{1}=v_{2}=\varphi(x)+\varepsilon \omega(x) \psi^{\varepsilon}(x)
$$

as the test functions in (2.10) where $\varphi, \omega \in \mathcal{D}(\Omega), \psi \in H_{p e r}^{1}(Y)$ and $\psi^{\varepsilon}(x)=$ $\psi(x / \varepsilon)$. This makes the integral on the interface vanish, so that we only have to treat the volume integrals.

By similar arguments as in the proof of Theorem 5.1, we get (5.8)-(5.12) where $\Phi_{1}$ and $\Phi_{2}$ are replaced by $\Phi(x, y)=\omega(x) \psi(y)$.

Hence, passing to the limit as $\varepsilon \rightarrow 0$ in the variational formulation (2.10) with the above choice of $\left(v_{1}, v_{2}\right)$, by density we deduce

$$
\begin{aligned}
& \frac{1}{|Y|} \sum_{i=1,2} \int_{\Omega \times Y_{i}} A(y)\left(\nabla u_{1}+\nabla_{y} \widehat{u}_{i}\right)\left(\nabla \varphi+\nabla_{y} \Phi\right) d x d y \\
& \quad+\frac{1}{|Y|} \sum_{i=1,2} \int_{\Omega \times Y_{i}} h_{i}\left(y, u_{1}\right) \varphi d x d y \\
& \quad=\int_{\Omega} f(x) \varphi(x) d x \quad \forall \varphi \in H_{0}^{1}(\Omega), \forall \Phi \in L^{2}\left(\Omega, H_{p e r}^{1}(Y)\right) .
\end{aligned}
$$

Let us now recall that from Remark 4.4 the relationship between the traces of $\widehat{u}_{1}$ and $\bar{u}_{2}$ on $\Gamma$ is given by $\widehat{u}_{1}=\widehat{u}_{2}+\xi_{\Gamma}$ on $\Omega \times \Gamma$. Then, the extension by periodicity of the function $\widehat{u}$ defined by (5.41) is a function (still denoted by $\widehat{u})$ which belongs to $L^{2}\left(\Omega, H_{\text {per }}^{1}(Y)\right)$ and satisfies

$$
\left.\nabla_{y} \widehat{u}\right|_{\Omega \times Y_{1}}=\nabla_{y} \widehat{u}_{1},\left.\quad \nabla_{y} \widehat{u}\right|_{\Omega \times Y_{2}}=\nabla_{y} \widehat{u}_{2} .
$$

Consequently, (5.43) gives the limit problem (5.42). 
Finally, the uniqueness of $\left(u_{1}, \widehat{u}_{1}, \widehat{u}_{2}\right)$, which implies the convergences of the whole sequence in (5.1), comes from the next corollary.

Corollary 5.7. For $\gamma<-1$, under the hypotheses of Theorem 5.6, let $u^{\varepsilon}=$ $\left(u_{1}^{\varepsilon}, u_{2}^{\varepsilon}\right)$ be the solution of problem (2.2). Then, there exists $u_{1} \in H_{0}^{1}(\Omega)$ such that, for $i=1,2$

$$
\begin{cases}\widetilde{u}_{i}^{\varepsilon} \rightarrow \theta_{i} u_{1} & \text { weakly in } L^{2}(\Omega), \\ A^{\varepsilon} \widetilde{\nabla u_{i}^{\varepsilon}} \rightarrow A_{\gamma}^{i} \nabla u_{1} & \text { weakly in }\left(L^{2}(\Omega)\right)^{n},\end{cases}
$$

$A_{\gamma}^{1}$ and $A_{\gamma}^{2}$ being the constant matrices defined by

$$
A_{\gamma}^{1} e_{j}=\theta_{1} \mathcal{M}_{Y_{1}}\left(A e_{j}-A \nabla \bar{\chi}_{j}\right), \quad A_{\gamma}^{2} e_{j}=\theta_{2} \mathcal{M}_{Y_{2}}\left(A e_{j}-A \nabla \bar{\chi}_{j}\right)
$$

where $\left\{e_{j}\right\}_{n}$ is the canonical basis of $\mathbb{R}^{n}$ and the correctors $\bar{\chi}_{j}, j=1, \ldots, n$, are the unique solutions of the cell problems

$$
\left\{\begin{array}{l}
-\operatorname{div}\left(A(y) \nabla\left(\bar{\chi}_{j}-y_{j}\right)\right)=0 \quad \text { in } Y \\
\bar{\chi}_{j} Y \text {-periodic, } \mathcal{M}_{\Gamma}\left(\bar{\chi}_{j}\right)=0 .
\end{array}\right.
$$

The function $u_{1}$ is the unique solution of the following problem:

$$
\left\{\begin{array}{l}
-\operatorname{div}\left(A_{\gamma}^{0} \nabla u_{1}\right)+\theta_{1} \mathcal{M}_{Y_{1}}\left(h_{1}\left(\cdot, u_{1}\right)\right)+\theta_{2} \mathcal{M}_{Y_{2}}\left(h_{2}\left(\cdot, u_{1}\right)\right)=f \quad \text { in } \Omega, \\
u_{1}=0 \text { on } \partial \Omega
\end{array}\right.
$$

where the homogenized matrix $A_{\gamma}^{0}$ is given by

$$
A_{\gamma}^{0} e_{j}=\mathcal{M}_{Y}\left(A e_{j}-A \nabla \bar{\chi}_{j}\right)=A_{\gamma}^{1} e_{j}+A_{\gamma}^{2} e_{j}
$$

Proof. Convergences (5.44) can be proved similarly as in [26].

Choosing $\varphi \equiv 0$ in (5.42), we get

$$
\frac{1}{|Y|} \int_{\Omega \times Y} A(y)\left(\nabla u_{1}+\nabla_{y} \widehat{u}\right) \nabla_{y} \Phi d x d y=0
$$

which implies that

$$
\left\{\begin{array}{l}
\operatorname{div}_{y}\left[A(y)\left(\nabla_{y} \widehat{u}(x, y)+\nabla u_{1}(x)\right)\right]=0 \text { a.e. in } \Omega \times Y, \\
\widehat{u}(x, \cdot) \quad Y \text {-periodic. }
\end{array}\right.
$$

Consequently by standard arguments, one obtains

$$
\widehat{u}(x, y)=-\sum_{j=1}^{n} \frac{\partial u_{1}}{\partial x_{j}}(x) \bar{\chi}_{j}(y),
$$

where $\bar{\chi}_{j}, j=1, \ldots, n$, are the solutions of the cell problems (5.45).

Now we choose $\Phi \equiv 0$ in (5.42),

$$
\begin{aligned}
& \frac{1}{|Y|} \int_{\Omega \times Y} A(y)\left(\nabla u_{1}+\nabla_{y} \widehat{u}\right) \nabla \varphi d x d y+\frac{1}{|Y|} \int_{\Omega \times Y_{1}} h_{1}\left(y, u_{1}\right) \varphi d x d y \\
& \quad+\frac{1}{|Y|} \int_{\Omega \times Y_{2}} h_{2}\left(y, u_{1}\right) \varphi d x d y=\int_{\Omega} f(x) \varphi(x) d x,
\end{aligned}
$$


that gives

$$
\left\{\begin{array}{l}
-\frac{1}{|Y|} \operatorname{div}\left(\int_{Y} A(y)\left[\nabla u_{1}(x)+\nabla_{y} \widehat{u}(x, y)\right] d y\right) \\
+\frac{1}{|Y|} \int_{Y_{1}} h_{1}\left(y, u_{1}(x)\right) d y+\frac{1}{|Y|} \int_{Y_{2}} h_{2}\left(y, u_{1}(x)\right) d y=f \quad \text { in } \Omega \\
u_{1}=0 \text { on } \partial \Omega .
\end{array}\right.
$$

Replacing (5.48) yields (5.46). The uniqueness of the solution $u_{1}$ of problem (5.46) is straightforward.

Remark 5.8. It is easy to check from the proof that for this case, we only need assumption $\left(\mathcal{H}_{2}^{\prime}\right)$ defined in Sect 3 , instead of $\left(\mathcal{H}_{2}\right)$, to obtain the homogenization result.

\subsection{The case $\gamma \in]-1,1]$}

Let us turn to the last case, where we state the results for $\gamma \in]-1,1[$ and $\gamma=1$ separately.

Theorem 5.9. Assume that $\gamma \in]-1,1\left[\right.$ and that $(2.4)-(2.6)_{1}$ hold. If $u^{\varepsilon}=$ $\left(u_{1}^{\varepsilon}, u_{2}^{\varepsilon}\right)$ is the solution of problem $(2.2)$, then there exist $u_{1} \in H_{0}^{1}(\Omega)$ and $\widehat{u}_{1} \in L^{2}\left(\Omega, H_{\text {per }}^{1}\left(Y_{1}\right)\right)$ such that

$$
\begin{cases}\mathcal{T}_{1}^{\varepsilon}\left(u_{1}^{\varepsilon}\right) \longrightarrow u_{1} & \text { strongly in } L^{2}\left(\Omega, H^{1}\left(Y_{1}\right)\right), \\ \mathcal{T}_{2}^{\varepsilon}\left(u_{2}^{\varepsilon}\right) \longrightarrow u_{1} & \text { strongly in } L^{2}\left(\Omega, H^{1}\left(Y_{2}\right)\right), \\ \mathcal{T}_{1}^{\varepsilon}\left(\nabla u_{1}^{\varepsilon}\right) \rightarrow \nabla u_{1}+\nabla_{y} \widehat{u}_{1} & \text { weakly in } L^{2}\left(\Omega \times Y_{1}\right), \\ \mathcal{T}_{2}^{\varepsilon}\left(\nabla u_{2}^{\varepsilon}\right) \rightarrow 0 & \text { weakly in } L^{2}\left(\Omega \times Y_{2}\right),\end{cases}
$$

and the pair $\left(u_{1}, \widehat{u}_{1}\right)$ is the unique solution of the problem

$$
\left\{\begin{array}{l}
\text { Find } u_{1} \in H_{0}^{1}(\Omega), \widehat{u}_{1} \in L^{2}\left(\Omega, W_{\text {per }}\left(Y_{1}\right)\right) \text { such that, } \\
\quad \frac{1}{|Y|} \int_{\Omega \times Y_{1}} A(y)\left(\nabla u_{1}+\nabla_{y} \widehat{u}_{1}\right)\left(\nabla \varphi+\nabla_{y} \Phi_{1}\right) d x d y \\
\quad+\frac{1}{|Y|} \sum_{i=1,2} \int_{\Omega \times Y_{i}} h_{i}\left(y, u_{1}\right) \varphi d x d y \\
\quad=\int_{\Omega} f(x) \varphi(x) d x, \forall \varphi \in H_{0}^{1}(\Omega), \forall \Phi_{1} \in L^{2}\left(\Omega, H_{p e r}^{1}\left(Y_{1}\right)\right) .
\end{array}\right.
$$

Proof. Theorems 4.3 and 4.5 imply convergences $(5.50)_{1-3}$ and

$$
\mathcal{T}_{2}^{\varepsilon}\left(\nabla u_{2}^{\varepsilon}\right) \rightarrow \nabla_{y} \bar{u}_{2} \quad \text { weakly in } L^{2}\left(\Omega \times Y_{2}\right)
$$

for some $\bar{u}_{2} \in L^{2}\left(\Omega, H^{1}\left(Y_{2}\right)\right)$, up to a subsequence.

We now choose as test functions in (2.10) the functions $v_{i}(x)=\varphi(x)+$ $\varepsilon \omega_{i}(x) \psi_{i}^{\varepsilon}(x), i=1,2$, where $\varphi, \omega_{i} \in \mathcal{D}(\Omega)$ and $\psi_{i}^{\varepsilon}(x)=\psi_{i}\left(\frac{x}{\varepsilon}\right)$, with $\psi_{1} \in$ $H_{\text {per }}^{1}\left(Y_{1}\right)$ and $\psi_{2} \in H^{1}\left(Y_{2}\right)$ (extended by $Y$-periodicity to $\mathbb{R}^{n}$ ). Arguing as in the proof of Theorem 5.1 gives convergences (5.10)-(5.12). 
Now we treat the nonlinear jump on the interface. Taking into account Remark 4.8, Proposition $4.2(i x)$ and the fact that the function $h$ satisfies assumption $\left(\mathcal{H}_{2}\right)$, we have

$$
\begin{aligned}
\varepsilon^{\gamma+1} & \int_{\Gamma^{\varepsilon}} h^{\varepsilon}\left(x, u_{1}^{\varepsilon}-u_{2}^{\varepsilon}\right)\left(v_{1}-v_{2}\right) d \sigma_{x} \\
= & \frac{\varepsilon^{\gamma+1}}{|Y|} \int_{\Omega \times \Gamma} h\left(y, \frac{\mathcal{T}_{b}^{\varepsilon}\left(u_{1}^{\varepsilon}-u_{2}^{\varepsilon}\right)}{\varepsilon}\right)\left(\psi_{1}(y) \mathcal{T}_{1}^{\varepsilon}\left(\omega_{1}\right)-\psi_{2}(y) \mathcal{T}_{2}^{\varepsilon}\left(\omega_{2}\right)\right) d x d \sigma_{y} \\
\leq & c \varepsilon^{\gamma+1}\left\|h\left(y, \frac{\mathcal{T}_{b}^{\varepsilon}\left(u_{1}^{\varepsilon}-u_{2}^{\varepsilon}\right)}{\varepsilon}\right)\right\|_{L^{\frac{2}{q}}(\Omega \times \Gamma)} \| \psi_{1}(y) \mathcal{T}_{1}^{\varepsilon}\left(\varphi_{1}\right) \\
& -\psi_{2}(y) \mathcal{T}_{2}^{\varepsilon}\left(\varphi_{2}\right) \|_{L^{\left(\frac{2}{q}\right)^{\prime}}(\Omega \times \Gamma)} \\
\leq & c \varepsilon^{\gamma+1}\left(1+\varepsilon^{-q}\left\|\mathcal{T}_{b}^{\varepsilon}\left(u_{1}^{\varepsilon}-u_{2}^{\varepsilon}\right)\right\|_{L^{2}(\Omega \times \Gamma)}^{q}\right) \\
\leq & c \varepsilon^{\gamma+1}\left(1+\varepsilon^{-q / 2}\left\|u_{1}^{\varepsilon}-u_{2}^{\varepsilon}\right\|_{L^{2}\left(\Gamma^{\varepsilon}\right)}^{q}\right) \\
\leq & c \varepsilon^{\gamma+1}\left(1+\varepsilon^{-(1+\gamma) q / 2}\right) \leq c\left(\varepsilon^{\gamma+1}+\varepsilon^{\frac{1}{2}(\gamma+1)(2-q)}\right) \longrightarrow 0,
\end{aligned}
$$

since $\gamma>-1$ and $q<2$.

From (5.10) to (5.12) and (5.53), passing to the limit as $\varepsilon \rightarrow 0$ in the variational formulation $(2.10)$ for $\left(v_{1}, v_{2}\right)$ given as above, we get

$$
\left\{\begin{array}{l}
\forall \varphi \in H_{0}^{1}(\Omega), \forall\left(\Phi_{1}, \Phi_{2}\right) \in L^{2}\left(\Omega, H_{p e r}^{1}\left(Y_{1}\right)\right) \times L^{2}\left(\Omega, H^{1}\left(Y_{2}\right)\right), \\
\frac{1}{|Y|} \int_{\Omega \times Y_{1}} A(y)\left(\nabla u_{1}+\nabla_{y} \widehat{u}_{1}\right)\left(\nabla \varphi+\nabla_{y} \Phi_{1}\right) d x d y \\
\quad+\frac{1}{|Y|} \sum_{i=1,2} \int_{\Omega \times Y_{i}} h_{i}\left(y, u_{1}\right) \varphi d x d y \\
\quad+\frac{1}{|Y|} \int_{\Omega \times Y_{2}} A(y) \nabla_{y} \bar{u}_{2}\left(\nabla \varphi+\nabla_{y} \Phi_{2}\right) d x d y=\int_{\Omega} f(x) \varphi(x) d x .
\end{array}\right.
$$

As in the proof of Theorem 3.3 in [26], choosing $\varphi=\Phi_{1} \equiv 0$ and $\Phi_{2} \equiv 0$ gives that $\nabla_{y} \bar{u}_{2}=0$ in $\Omega \times Y_{2}$. Hence, convergence $(5.50)_{4}$ follows from (5.52). Finally, the uniqueness of $\left(u_{1}, \widehat{u}_{1}\right)$ which implies the convergences of the whole sequence in (5.50) comes from the next corollary. following:

By similar arguments to those used in Theorem 5.7, we can prove the

Corollary 5.10. For $\gamma \in]-1,1[$, assume that the hypotheses of Theorem 5.9 are satisfied and $u^{\varepsilon}=\left(u_{1}^{\varepsilon}, u_{2}^{\varepsilon}\right)$ is the solution of problem (2.2). Then there exists $u_{1} \in H_{0}^{1}(\Omega)$ such that

$$
\begin{cases}\widetilde{u}_{i}^{\varepsilon} \rightarrow \theta_{i} u_{1} & \text { weakly in } L^{2}(\Omega), i=1,2, \\ A^{\varepsilon} \widetilde{\nabla u_{1}^{\varepsilon}} \rightarrow A_{\gamma}^{0} \nabla u_{1} & \text { weakly in }\left(L^{2}(\Omega)\right)^{n}, \\ A^{\varepsilon} \widehat{\nabla u_{2}^{\varepsilon}} \rightarrow 0 & \text { weakly in }\left(L^{2}(\Omega)\right)^{n},\end{cases}
$$


and $u_{1}$ is the unique solution of problem (5.46), where the homogenized matrix $A_{\gamma}^{0}$ is the constant matrix defined by

$$
A_{\gamma}^{0} e_{j}=\theta_{1} \mathcal{M}_{Y_{1}}\left(A e_{j}-A \nabla \chi_{j}\right)
$$

The correctors $\chi_{j}, j=1, \ldots, n$, are the unique solutions of the cell problems

$$
\begin{cases}-\operatorname{div}\left(A(y) \nabla\left(\chi_{j}-y_{j}\right)\right)=0 & \text { in } Y_{1}, \\ A(y) \nabla\left(\chi_{j}-y_{j}\right) \cdot n_{1}=0 & \text { on } \Gamma, \\ \chi_{j} Y \text {-periodic, } \mathcal{M}_{\Gamma}\left(\chi_{j}\right)=0 . & \end{cases}
$$

Let us now state the homogenization result for the case $\gamma=1$, where we make use of the same test functions and similar arguments in the proof of Theorem 5.9.

Theorem 5.11. Let $\gamma=1$ and the assumptions (2.4)-(2.6) $)_{3}$ hold. If $u^{\varepsilon}=$ $\left(u_{1}^{\varepsilon}, u_{2}^{\varepsilon}\right)$ is the solution of problem $(2.2)$, then there exist $u_{1} \in H_{0}^{1}(\Omega)$ and $\widehat{u}_{1} \in$ $L^{2}\left(\Omega, H_{p e r}^{1}\left(Y_{1}\right)\right)$ such that convergences $(5.50)_{1,3,4}$ hold and $\left(u_{1}, \widehat{u}_{1}\right)$ uniquely satisfies

$$
\left\{\begin{array}{l}
u_{1} \in H_{0}^{1}(\Omega), \widehat{u}_{1} \in L^{2}\left(\Omega, H_{p e r}^{1}\left(Y_{1}\right)\right) \text { with } \mathcal{M}_{\Gamma}\left(\widehat{u}_{1}\right)=0 \text { a.e. } x \in \Omega, \\
\quad+\frac{1}{|Y|} \int_{\Omega \times Y_{1}} A(y)\left(\nabla u_{1}+\nabla_{y} \widehat{u}_{1}\right)\left(\nabla \varphi+\nabla_{y} \Phi_{1}\right) d x d y \\
\quad h_{1}\left(y, u_{1}\right) \varphi d x d y \\
\quad=\int_{\Omega} f(x) \varphi(x) d x, \forall \varphi \in H_{0}^{1}(\Omega), \forall \Phi_{1} \in L^{2}\left(\Omega, H_{p e r}^{1}\left(Y_{1}\right)\right) .
\end{array}\right.
$$

Moreover, the homogenized problem is the following:

$$
\left\{\begin{array}{l}
-\operatorname{div}\left(A_{\gamma}^{0} \nabla u_{1}\right)+\theta_{1} \mathcal{M}_{Y_{1}}\left(h_{1}\left(\cdot, u_{1}\right)\right)=f \quad \text { in } \Omega, \\
u_{1}=0 \text { on } \partial \Omega
\end{array}\right.
$$

where $A_{\gamma}^{0}$ is defined by (5.55).

Remark 5.12. When $\gamma=1$, Theorem 4.3 only provides the weak convergence of $\mathcal{T}_{2}^{\varepsilon}\left(u_{2}^{\varepsilon}\right)$ to the function $u_{2}$ in $L^{2}\left(\Omega ; H^{1}\left(Y_{2}\right)\right)$, so that we are not able to characterize $u_{2}$, except when $h$ is linear as in [26]. In that case, this is done by choosing $v_{1}=0$ and $v_{2} \in \mathcal{D}(\Omega)$ as test functions in the variational formulation and passing to the limit as $\varepsilon \rightarrow 0$. To do that, the weak convergence above is sufficient since $\varepsilon h\left(y, \mathcal{T}_{b}^{\varepsilon}\left(u_{1}^{\varepsilon}-u_{2}^{\varepsilon}\right) / \varepsilon\right)$ is of the form $\widetilde{h}(y) \mathcal{T}_{b}^{\varepsilon}\left(u_{1}^{\varepsilon}-u_{2}^{\varepsilon}\right)$. This is not the case anymore when $h$ is not linear then here we cannot prove the uniqueness of $u_{2}$. Consequently, the convergence of $\mathcal{T}_{2}^{\varepsilon}\left(u_{2}^{\varepsilon}\right)$ in $(4.2)_{1}$ holds only for a subsequence.

Observe also that the assumption $h_{2} \equiv 0$ is motivated by the fact that Theorem 4.5 does not apply for $\gamma=1$, so that one cannot pass to the limit in the unfolded term concerning $h_{2}$. The same assumption has been used by 
Timofte in [41] for the case where the domain $\Omega_{2}^{\varepsilon}$ is also connected. The case $h_{2} \not \equiv 0$ remains an open problem.

\section{Acknowledgements}

The authors are deeply grateful to Doina Cioranescu, Alain Damlamian and Georges Griso for useful comments and discussions. The second author wishes to express her deep gratitude to Ministry of Education and Training of Vietnam for its financial support, which made this work possible.

\section{References}

[1] Allaire, G.: Homogenization and two-scale convergence. SIAM J. Math. Anal. 23, 1482-1518 (1992)

[2] Amar, M., Andreucci, D., Gianni, R.: Evolution and memory effects in the homogenization limit for electrical conduction in biological tissues. Math. Model. Methods Appl. Sci. 14, 1261-1295 (2004)

[3] Amar, M., Andreucci, D., Bisegna, P., Gianni, R.: A hierarchy of models for the electrical conduction in biological tissues via two-scale convergence: The nonlinear case. Differ. Integral Equations 26(9-10), 885-912 (2013)

[4] Auriault, J.L., Ene, H.: Macroscopic modelling of heat transfer in composites with interfacial thermal barrier. Int. J. Heat Mass Tranfer 37, 2885-2892 (1994)

[5] Barenblatt, G.I., Zheltov, Y.P., Kochina, I.N.: Basic concepts in the theory of seepage of homogeneous liquids in fractured rocks. Prikl. Mat. Mekh. 24(5), 852$864(1960)$

[6] Bensoussan, A.; Lions, J.-L.; Papanicolaou, G: Asymptotic Analysis for Periodic Structures. North Holland, Amsterdam (1978)

[7] Cabarrubias, B., Donato, P.: Existence and uniqueness for a quasilinear elliptic problem with nonlinear Robin conditions. Carpathian J. Math. 27(2), 173$184(2011)$

[8] Cabarrubias, B., Donato, P.: Homogenization of a quasilinear elliptic problem with nonlinear Robin boundary conditions. Appl. Anal. 91(6), 1111-1127 (2011)

[9] Canon, E., Pernin, J.N.: Homogénéisation d'un problème de diffusion en milieu composite avec barrière à l'interface. C. R. Acad. Sci., Ser. I, Math. 325(1), 123$126(1997)$

[10] Carslaw, H.S., Jaeger, J.C.: Conduction of Heat in Solids. At the Clarendon Press, Oxford (1959)

[11] Cioranescu D., Damlamian, A., Griso, G.: Periodic unfolding and homogenization. C. R. Acad. Sci. Paris, Série 1, 335, 99-104 (2002)

[12] Cioranescu, D., Damlamian, A., Griso, G.: The periodic unfolding method in homogenization. SIAM J. Math. Anal. 40(4), 1585-1620 (2008) 
[13] Cioranescu, D., Damlamian, A., Donato, P., Griso, G., Zaki, R.: The periodic unfolding method in domains with holes. SIAM J. Math. Anal. 44(2), 718$760(2012)$

[14] Cioranescu, D., Donato, P.: An Introduction to Homogenization, Oxford Lecture Series in Mathematics and Its Applications, vol. 17 (1999)

[15] Cioranescu, D., Donato, P., Zaki, R.: The periodic unfolding method in perforated domains. Portugaliae Math. 63(4), 467-496 (2006)

[16] Cioranescu, D., Donato, P., Zaki, R.: Asymptotic behavior of elliptic problems in perforated domains with nonlinear boundary conditions. Asymptot. Anal. 53(4), 209-235 (2007)

[17] Cioranescu, D., Saint Jean Paulin, J.: Homogenization open sets with holes. J. Math. Anal. Appl. 71, 590-607 (1979)

[18] Cioranescu, D., Paulin, J.S.J.: Homogenization of reticulated structures. In: Appl. Math. Sci., vol. 139. Springer, New York (1999)

[19] Damlamian, A., Meunier, N., Van Schaftingen, J.: Periodic homogenization of monotone multivalued operators. Nonlinear Anal. 67, 3217-3239 (2007)

[20] Donato, P.: Some corrector results for composites with imperfect interface. Rend. Math. Ser. VII 26, 189-209 (2006)

[21] Donato, P.: Homogenization of a class of imperfect transmission problems. In: Damlamian, A., Miara, B., Li, T. (eds.) Multiscale Problems: Theory, Numerical Approximation and Applications. Series in Contemporary Applied Mathematics CAM, vol. 16, pp. 109-147. Higher Education Press, Beijing (2011)

[22] Donato, P., Faella, L., Monsurrò, S.: Homogenization of the wave equation in composites with imperfect interface: a memory effect. J. Math. Pures Appl. 87, 119-143 (2007)

[23] Donato, P., Faella, L., Monsurrò, S.: Correctors for the homogenization of a class of hyperbolic equations with imperfect interfaces. SIAM J. Math. Anal. 40(5), 1952-1978 (2009)

[24] Donato, P., Jose, E.C.: Corrector results for a parabolic problem with a memory effect. ESAIM Math. Model. Numerical Anal. 44, 421-454 (2010)

[25] Donato, P., Monsurrò, S.: Homogenization of two heat conductors with an interfacial contact resistance. Anal. Appl. 2(3), 247-273 (2004)

[26] Donato, P., Le Nguyen, K.H., Tardieu, R.: The periodic unfolding method for a class of imperfect transmission problems. J. Math. Sci. 176(6), 891-927 (2011)

[27] Ene, H.I.: On the microstructure models of porous media. Rev. Roum. Math. Pures Appl. 46(2-3), 289-295 (2001)

[28] Ene, H.I., Poliševski, D.: Model of diffusion in partially fissured media. ZAMP 53, 1052-1059 (2002) 
[29] Faella, L., Monsurrò, S: Memory effects arising in the homogenization of composites with inclusions. In: Topics on Mathematics for Smart Systems, pp. 107-121. World Sci. Publ, Hackensack (2007)

[30] Hummel, H.K.: Homogenization for heat tranfer in polycrystals with interfacial resistances. Appl. Anal. 75(3-4), 403-424 (2000)

[31] Jose, E.C.: Homogenization of a parabolic problem with an imperfect interface. Rev. Roum. Math. Pures Appl. 54(3), 189-222 (2009)

[32] Krasnosel'skii, MA.: Topological Methods in the theory of nonlinear intergral equations. In: International Series of Monographs in Pure and Applied Mathematics. Pergamon Press, New York (1964)

[33] Lipton, R.: Heat conduction in fine scale mixtures with interfacial contact resistance. Siam J. Appl. Math. 58(1), 55-72 (1998)

[34] Lipton, R., Vernescu, B.: Composite with imperfect interface. Proc. Soc. Lond. A 452, 329-358 (1996)

[35] Mardare, S.: Personal communication

[36] Monsurrò, S.: Homogenization of a two-component composite with interfacial thermal barrier. Adv. Math. Sci. Appl. 13(1), 43-63 (2003)

[37] Monsurrò, S.: Erratum for the paper homogenization of a two-component composite with interfacial thermal barrier. Adv. Math. Sci. Appl. 14, 375-377 (2004)

[38] Nguetseng, G.: A general convergence result for a functional related to the theory of homogenization. SIAM J. Math. Anal. 20, 608-629 (1989)

[39] Pernin, J.N.: Homogénéisation d'un problème de diffusion en milieu composite à deux composantes. C.R. Acad. Sci. Paris Série I, 321, 949-952 (1995)

[40] Tartar, L.: Quelques remarques sur l'homogénéisation. In: Functional Analysis and Numerical Analysis, Proc. Japan-France Seminar 1976 (Fujita ed.), Japanese Society for the Promotion of Science, pp. 468-482 (1978)

[41] Timofte, C.: Upscaling in nonlinear diffusion problems in composite materials, Progress in Industrial Mathematics at ECMI (2006), 328-332

Patrizia Donato and Kim Hang Le Nguyen

Laboratoire de Mathématiques Raphaël Salem

CNRS UMR 6085

Normandie Université

Université de Rouen

Avenue de l'Université

BP 12

76801 Saint-Étienne du Rouvray Cedex

France

e-mail: patrizia.donato@univ-rouen.fr 
Kim Hang Le Nguyen

Faculty of Sciences

Nong Lam University

Linh Trung ward, Thu Duc district

Ho Chi Minh City

Vietnam

e-mail: lehangkim@gmail.com

Received: 3 May 2014.

Accepted: 11 April 2015. 